\title{
Recommandations de bonne pratique clinique concernant la prise en charge médicale des victimes d'une " tuerie de masse "
}

\author{
French Guidelines on Good Practice Concerning Medical Care of a Mass Casualty Incident
}

\author{
E. Cesareo $\cdot$ M. Raux $\cdot$ L. Soulat $\cdot$ F. Huot-Marchand $\cdot$ E. Voiglio $\cdot$ A. Puidupin - P.-G. Claret - J.-P. Desclef $\cdot$ \\ B. Douay · J. Duchenne · A. Gloaguen · H. Lefort · D. Rerbal · C. Zanker · F. Cook · G. Pelée de Saint Maurice · \\ L. Lachenaud $\cdot$ L. Gabilly $\cdot$ N. Prieto $\cdot$ J. Levraut $\cdot$ P.-Y. Gueugniaud
}

Reçu le 16 septembre 2018 ; accepté le 1 octobre 2018

(C) SFMU et Lavoisier SAS 2018
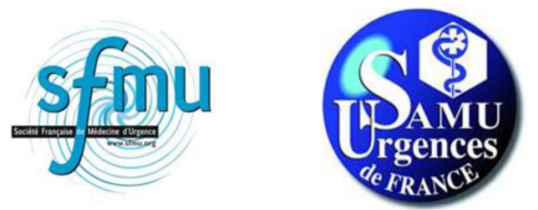
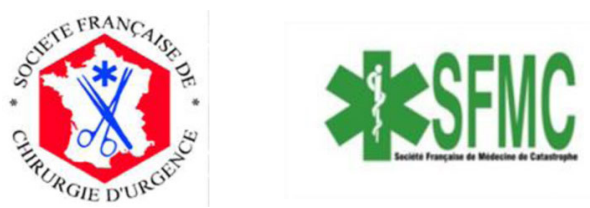

E. Cesareo $(\bowtie) \cdot$ D. Rerbal $\cdot$ L. Gabilly

Samu 69, hôpital Édouard-Herriot, hospices civils de Lyon, 5, place d'Arsonval, F-69437 Lyon cedex 03, France eric.cesareo@chu-lyon.fr

\section{Raux}

Inserm UMRS 1158,

département d'anesthésie-réanimation,

groupe hospitalier Pitié-Salpêtrière-Charles-Foix,

Assistance Publique-Hôpitaux de Paris (APHP),

Sorbonne Université, F-75013 Paris, France

\section{Soulat}

Samu 35-Smur-Urgences, CHU de Rennes, université Rennes-I, 2, rue Henri-Le-Guilloux,

F-35033 Rennes cedex 09, France

F. Huot-Marchand · L. Lachenaud

Société française de médecine de catastrophe (SFMC),

38, rue Dunois, F-75647 Paris cedex 13, France

\section{E. Voiglio}

Société française de chirurgie d'urgence,

Direction de l'action sanitaire, division de santé,

48, boulevard d'Italie, MC-98000 Monaco, Monaco

A. Puidupin

Service de santé des armées,

60, boulevard du Général Martial Valin

CS 21623, F-75509 Paris cedex 15, France

Direction générale de la santé,

14, avenue Duquesne F-75007 Paris, France

P.-G. Claret

Pôle anesthésie-réanimation-douleur-urgences,

CHU de Nîmes, F-30900 Nîmes, France

\section{J.-P. Desclef}

Samu 91, Smur Corbeil, centre hospitalier Sud-Francilien, 40, avenue Serge-Dassault, F-91100 Corbeil-Essonnes, France
B. Douay

Département de médecine d'urgence,

centre hospitalier universitaire Amiens-Picardie,

F-80054 Amiens cedex 01, France

J. Duchenne

Pôle d'addictologie et de médecine d'urgence,

centre hospitalier Henri-Mondor, 50, avenue de la République, F-15002 Aurillac cedex, France

\section{A. Gloaguen}

Centre régional universitaire des urgences,

centre hospitalier universitaire de Dijon-Bourgogne,

14, rue Gaffarel, F-21000 Dijon, France

\section{H. Lefort}

Structure des urgences, hôpital d'instruction des armées

Legouest, BP 90001,

F-57077 Metz cedex 03, France

C. Zanker

Service des urgences, institut hospitalier Franco-Britannique,

4, rue Kléber, F-92300 Levallois, France

F. Cook

Service d'anesthésie et des réanimations chirurgicales, groupe hospitalo-universitaire Henri-Mondor-Albert-Chenevier, Assistance publique-Hôpitaux de Paris,

51, avenue du Maréchal-de-Lattre-de-Tassigny,

F-94010 Créteil cedex, France

G. Pelée de Saint Maurice

Département d'anesthésie-réanimation,

hôpital d'instruction des armées Percy,

F-92141 Clamart cedex, France

N. Prieto

Cellule d'urgence médicopsychologique, Samu,

hôpital Édouard-Herriot, hospices civils de Lyon,

5, place d'Arsonval,

F-69437 Lyon cedex 03, France 


\section{Rédigées avec les représentants de :}

Samu-Urgences de France (SUdF), la Société française d'anesthésie et de réanimation (Sfar), la Société française de chirurgie d'urgence (SFCU), la Société française de médecine de catastrophe (SFMC)

\section{En lien avec :}

Le Service de santé des armées (SSA)

La Direction générale de la santé (DGS)

\section{Groupe de relecture}

C. Ammirati (Amiens), S. Ausset (Clamart), F. Berthier (Nantes), C. Bertrand (Créteil), F. Braun (Metz), P. Carli (Paris), J.-S. David (Lyon), T. Gauss (Clichy), H. Julien (Paris), M. Langlois (Bièvres), E. Lecarpentier (Créteil), J.-M. Philippe (Paris), E. Querellou (Brest), A. Ricard Hibon (Pontoise), F. Rongieras (Lyon), K. Tazarourte (Lyon), J.-P. Tourtier (Saint-Mandé), M. Vergne (Toulon)

\section{Membres de la commission des référentiels de la SFMU}

E. Cesareo (Lyon), A. Chauvin (Paris), P.-G. Claret (Nîmes), J.-P. Desclefs (Corbeil-Essonnes), B. Douay (Amiens), J. Duchenne (Aurillac), A. Gloaguen (Dijon), P. Le Conte (Nantes), H. Lefort (Metz), M. Martinez (Montbrison), M. Oberlin (Cahors), D. Rerbal (Lyon), G. Valdenaire (Bordeaux), J. Vaux (Créteil), D. Viglino (Grenoble), C. Zanker (Levallois)

\section{Introduction}

L'expression « tuerie de masse », traduction approximative du terme anglais massive shooting, est utilisée dans toutes les circulaires ou instructions de l'État. Cependant, il n'existe aucune définition universelle d'une tuerie de masse. Nous nous proposons de définir la tuerie de masse comme " une agression volontaire, réalisée par un ou des individus sur un ou plusieurs sites, provoquant un nombre de victimes dont la prise en charge peut dépasser les moyens sanitaires immédiatement disponibles ». Quels que soient les moyens employés, il s'agit d'un acte hostile qui a le plus souvent pour but de terroriser l'opinion publique et de déstabiliser les structures de l'État [1].

Dans le rapport de l'Assemblée nationale fait au nom de la commission d'enquête relative " aux moyens mis en œuvre pour lutter contre le terrorisme depuis le 7 janvier 2015 », plusieurs intervenants ont déclaré qu'il fallait « nous préparer à être surpris » [2,3]. L'organisation de la réponse du système de santé est devenue aujourd'hui un enjeu majeur de santé publique [4]. Si l'élaboration de plans, la réalisation d'exercices sont indispensables, la diffusion de doctrines communes d'intervention et de prise en charge médicale l'est tout autant, afin d'apporter des niveaux de réponse adaptés à des agressions collectives $[2,5,6]$. C'est la raison pour laquelle le conseil d'administration de la Société française de médecine d'urgence (SFMU) a missionné sa commission des référentiels afin de rédiger des recommandations concernant la prise en charge médicale des victimes d'une « tuerie de masse ». Ces recommandations s'appuient sur une revue de la littérature médicale, des textes réglementaires et des avis d'experts. La littérature médicale concernant la prise en charge des victimes d'une tuerie de masse est limitée. Les publications reposent essentiellement sur des retours d'expérience et des avis d'experts. Afin que ces recommandations soient fondées sur un large consensus médical, la SFMU a souhaité associer des experts au titre de Samu-Urgences de France (SUdF), de la Société française d'anesthésie et de réanimation (Sfar), de la Société française de médecine de catastrophe (SFMC), de la Société française de chirurgie d'urgence (SFCU) et du Service de santé des armées (SSA). La méthode « Recommandations par consensus formalisé », publiée dans les " Recommandations pour la pratique clinique » éditées par la Haute Autorité de santé (HAS), a été retenue [7]. Ce texte aborde la prise en charge globale des victimes de façon chronologique aussi bien sur le versant extrahospitalier qu'intrahospitalier. La prise en charge médicale spécifique d'une attaque fondée sur un usage d'agents non conventionnels (nucléaire, radiologique, biologique et chimique) a été volontairement exclue du champ de ce référentiel.

\section{Gestion de l'alerte}

La précocité de l'alerte est capitale, car elle permet une anticipation de la crise. Elle doit être donnée dès connaissance 
par les services de secours et de soins d'urgences préhospitaliers d'un potentiel événement avec de nombreuses victimes. La mobilisation des établissements de santé identifiés comme étant de première ligne dans le volet AMAVI (accueil massif de victimes) du dispositif ORSAN (organisation de la réponse sanitaire) doit être immédiate dès l'alerte ou la notion de l'événement, sans attendre le déclenchement officiel du plan blanc de ces établissements [8,9].

\section{Réception au Samu-Centre 15 d'un appel évoquant une tuerie de masse}

Dès réception d'un ou plusieurs appels pouvant faire évoquer une tuerie de masse, il est impératif de recueillir les informations nécessaires à l'organisation médicale, mais aussi d'évaluer la menace afin de ne pas exposer les équipes de secours à des risques potentiels ou avérés [10]. Il est important de faire préciser par le ou les premiers témoins :

- la localisation géographique la plus précise possible ;

- le ou les agents vulnérants utilisés (arme à feu, explosion, véhicule bélier, gaz...) ;

- le nombre approximatif et le type de victimes (adultes, enfants) ;

- l'état présumé des victimes concernées.

Dans le même temps, des consignes sont données aux personnes sur place :

- s'éloigner, voire s'échapper si possible ;

- se cacher si la menace persiste, mettre son téléphone en mode silencieux et éviter de faire du bruit.

Le numéro téléphonique 114 habituellement réservé aux malentendants peut être utilisé par tous pour signaler par message texte une situation d'urgence aux forces de l'ordre [11].

\section{Actions immédiates à mener en régulation médicale}

L'interconnexion entre le Centre de réception et de régulation des appels d'urgence (CRRA) des Samu-Centres 15, le Centre d'information et de commandement des forces de police (CIC), le Centre d'opération et de renseignement de la gendarmerie (CORG) et le Centre de traitement de l'alerte du Centre opérationnel départemental d'incendie et de secours du Service d'incendie et de secours (CTA-CODIS du SIS) permet de croiser et de confirmer les informations sur la nature des faits et obtenir rapidement la description la plus précise possible de l'événement [12].

Une régulation médicale de crise est mise en place avec un médecin urgentiste expérimenté et des assistants de régulation médicale (ARM) dédiés. L'équipe constituée gère uniquement les appels émanant de l'attentat et coordonne les actions liées à cet événement dans un lieu spécifique dédié et anticipé (cellule de crise du Samu). Pour optimiser et adapter le fonctionnement de cette régulation de crise, un rappel immédiat d'ARM et de médecins en capacité de réguler une situation exceptionnelle doit être effectué.

Le médecin régulateur prévient le chef de service du Samu ou le médecin d'astreinte ainsi que le directeur de garde du centre hospitalier $(\mathrm{CH})$ siège du Samu. Il fait alerter les établissements de santé de première ligne et les experts identifiés dans le volet ORSAN AMAVI, notamment les centres de traumatologie de proximité et les structures d'urgence des établissements de soins impliqués dans la prise en charge habituelle des traumatisés. Il fait aussi alerter les autres établissements de santé situés à proximité de l'événement, qui peuvent être concernés par un afflux spontané de personnes blessées sans avoir toutefois un plateau technique adéquat. Cela doit permettre aux établissements de santé d'anticiper une configuration AMAVI (vérification de la conformité de leur flux capacitaire, arrêt de l'activité programmée dans le but d'accueillir un afflux de victimes et information du Samu sur toute difficulté conjoncturelle) [13]. Il prévient le Samu zonal, l'Agence régionale de santé (ARS) et s'assure que l'information a bien été transmise à l'autorité préfectorale.

La régulation médicale de crise recense les personnels présents et les moyens à disposition immédiate (unités mobiles hospitalières terrestres, moyens héliportés). Elle alerte les équipes Smur et, dans la mesure du possible, les ambulances privées. Les transferts secondaires non urgents sont différés. Une géolocalisation des vecteurs Smur départementaux constitue une plus-value nécessitant une réflexion menée en amont entre l'ARS et les établissements hospitaliers sièges du Samu [10].

Le risque de surattentat ou d'attaque multisites, omniprésent, est constamment à prendre en compte et à anticiper [14]. Ce risque intègre les dispositifs de secours comme les établissements de soins. Il impose un engagement réfléchi des moyens Smur et une sécurisation des établissements de santé (ES). Un rappel des consignes élémentaires de sécurité doit être fait aux équipes qui vont être engagées. Le port des équipements de protection individuels adaptés au contexte notamment nucléaire, radiologique, biologique, chimique et explosif (NRBCE) doit être anticipé. Une réflexion menée en amont entre l'autorité préfectorale en lien avec l'ARS, les autorités de police, de gendarmerie et l'autorité militaire de la région doit définir la force responsable, les objectifs et les moyens qui seront engagés pour assurer la sécurisation des ES. Cette réflexion doit être formalisée par une procédure engageant les différents acteurs.

\section{Actions une fois l'alerte confirmée}

La régulation médicale mobilise les moyens des plans de secours [15]. Les Samu, les centres de traumatologie et les services d'urgences doivent être dotés d'un système 
automatisé de rappel du personnel gradué, avec système d'acquittement de réception du message et de la réponse, directement intégré dans un logiciel. Dans le message de rappel, le lieu de rassemblement des personnels mobilisés doit être spécifié afin d'organiser l'acheminement, la traçabilité et l'affectation des équipes.

L'alerte avérée est transmise par le Samu à la fois aux établissements de santé de proximité pouvant être amenés à prendre en charge des patients et aux ES, en particulier les centres de traumatologie de la région conformément à la planification AMAVI connue de tous.

Le médecin régulateur du Samu doit s'enquérir auprès du CODIS de la localisation du point de passage obligé (PPO) et du point de regroupement des moyens (PRM). Le PRM est défini par le commandant des opérations de police et de gendarmerie (COPG) qui constitue la force menante à ce moment de l'alerte. Le COPG a la responsabilité de la sécurisation du PRM et de la sécurité des secours engagés [16]. Le directeur des secours médicaux (DSM) est désigné parmi les primointervenants médicaux engagés en attente du DSM inscrit sur la liste d'aptitude et désigné préalablement par le préfet (directeur des opérations [DO]) [12]. Ce qui impose dans le choix du médecin de la première équipe Smur engagée qu'il s'agisse du médecin disponible le plus expérimenté.

Les équipes médicales sont constituées, et en fonction de la dynamique de rappel, la disponibilité aux départs est anticipée. Les Smur, les Samu limitrophes et le Samu zonal sont mis en alerte ainsi que les vecteurs supplémentaires potentiellement disponibles. En fonction du lieu, un renfort héliporté doit être anticipé. Les moyens de communication de type portatif radio, téléphone satellite sont prêts à l'emploi. Les véhicules sont armés avec les lots prévus pour les postes sanitaires mobiles (lots PSM) et des kits damage control (DC) [adultes et pédiatriques]. Des kits supplémentaires selon la typologie des victimes (brûlés, intoxication, NRBC) sont mobilisés si besoin. Le véhicule PC Samu est activé. Les moyens du Smur territorial compétent sont engagés. La cellule d'urgence médicopsychologique (CUMP) est déclenchée.

En fonction des besoins de la chaîne médicale, les moyens médicaux présents au PRM sont engagés par le DSM et répartis sur site en concertation avec le commandant des opérations de secours (COS) et le COPG. L'ambulancier Smur, en lien avec l'officier sapeur-pompier responsable du PRM, dépose son équipe puis retourne au PRM stationner son véhicule afin de ne pas saturer l'accès au site. Les nouvelles informations influençant les décisions doivent être immédiatement répercutées entre services : Samu, sapeurspompiers et forces de sécurité intérieure (FSI). Dans la mesure du possible, désigner un ambulancier Smur avec pour mission prioritaire la gestion des vecteurs médicaux engagés au PRM est souhaitable.
En cas d'activité partagée du personnel médical et paramédical entre structures extra- et intrahospitalières, une réflexion au sein de chaque unité doit être menée en amont afin de définir une répartition adaptée à l'événement et aux compétences de chacun. Elle fera l'objet d'une procédure écrite annexée au règlement intérieur du Samu-Smur et de la structure des urgences.

Au moment de la crise, les équipes immédiatement disponibles doivent être engagées en économisant au maximum les moyens opérationnels pour répondre aux urgences quotidiennes. Il faut garder à l'esprit un possible surattentat ou des sites multiples. Anticiper la gestion logistique du personnel médical et non médical lors de ces événements est indispensable (personnels à rappeler non postés dans les six heures qui suivent, reconstitution d'équipes pour la gestion des affaires courantes).

\section{Gestion initiale de l'alerte en intrahospitalier}

Un capacitaire théorique en cas d'afflux de victimes doit avoir été défini à l'avance entre l'ARS et chaque établissement de santé impliqué dans le volet AMAVI du dispositif ORSAN (établissements de santé de première ligne, experts et de replis). La connaissance de ces flux capacitaires est connue par les acteurs concernés (délégation territoriale de l'agence régionale de santé (DTARS), Samu départemental, Samu de la zone de défense et ES). La prise en charge des urgences pédiatriques doit faire l'objet d'une réflexion préalable identique. Les ES s'engagent par la suite à mettre en place les moyens nécessaires en cas de déclenchement du volet AMAVI du dispositif ORSAN pour assurer ce flux capacitaire qui doit, en particulier pour les urgences absolues (UA), être envisagé en fonction de la capacité qu'ont les établissements à libérer immédiatement ou dans l'heure des salles de bloc opératoire. L'utilisation d'outils informatiques de gestion des blocs opératoires facilite la simulation d'un capacitaire d'accueil selon le jour et l'heure.

Les établissements de santé doivent convenir avec le Samu départemental d'un numéro téléphonique unique, dûment identifié, disponible 24 heures sur 24 et 7 jours sur 7 . Selon l'établissement, ce numéro peut être identifié au sein des services des urgences, des services d'accueil déchocage ou des unités de réanimation. L'interlocuteur recevant l'alerte doit être un médecin senior. Il doit connaître la procédure de mise en œuvre du volet ORSAN AMAVI du plan blanc et être capable de l'initier au sein de son service et de son établissement en attendant l'arrivée du directeur médical de crise (DMC). Le DMC est le conseiller médical du directeur d'établissement pour la gestion de la crise [17]. Il est également le correspondant médical de l'établissement auprès du Samu. Ses fonctions sont développées dans le paragraphe Direction médicale intrahospitalière. Il doit être facilement identifiable, notamment par le port d'une chasuble. 
À la réception de l'alerte, le DMC doit être en mesure de confirmer immédiatement au Samu que son établissement ne subit pas de circonstance exceptionnelle l'empêchant de satisfaire au flux capacitaire d'accueil prédéfini. Il sait si son établissement est en mesure d'accueillir des victimes pédiatriques dans les minutes ou les heures immédiates qui suivent le début de la crise. Le lieu, l'ampleur et le caractère confirmé ou non de la situation sanitaire exceptionnelle (SSE) doivent être renseignés pour permettre au DMC de prendre les décisions proportionnées à l'événement [13].

Un circuit d'alerte interne doit avoir été prévu pour activer rapidement les cellules de pilotage des secteurs clés (structure de médecine d'urgence, déchocage, salle de surveillance postinterventionnelle (SSPI) et blocs opératoires, réanimations, service des admissions, direction d'établissement).

Un recensement du capacitaire d'aval des structures d'accueil des UA et des urgences relatives (UR) est initié pour préparer leur mise en condition AMAVI. Un recensement de l'activité des blocs opératoires est réalisé. Un recensement des personnels médicaux présents est initié (urgentistes, anesthésistes-réanimateurs, réanimateurs médicaux, chirurgiens, radiologues) pour optimiser les rappels de personnel. Ces recensements doivent être réalisés par des acteurs identifiés à l'avance par le dispositif AMAVI selon une procédure écrite permettant de décharger le médecin de cette tâche. L'utilisation de fiches actions et de tableaux préremplis est recommandée. Les informations résultantes seront centralisées par le DMC.

\section{Montée en puissance des établissements de santé}

La montée en puissance doit intervenir dès confirmation de la SSE et aboutir dans l'heure qui suit l'alerte à la mise en configuration AMAVI de l'établissement [18]. L'alerte est répercutée selon une procédure établie dans le plan blanc, sur l'ensemble des services ressources de l'établissement : radiologie, laboratoires, Établissement français du sang (EFS), stérilisation, pharmacie, sécurité, transports internes des patients, direction des services informatiques, standard téléphonique, restauration, crèches... Chaque service doit posséder une procédure interne de mise en configuration AMAVI [17].

Les cellules de pilotage internes sont formées :

- cellule d'accueil des UA ;

- cellule d'accueil des UR ;

- cellule bloc opératoire ;

- cellule des admissions ;

- cellule de crise de la direction d'établissement ;

- cellule de prise en charge intrahospitalière des urgences médicopsychologiques en lien avec la CUMP.

Ces cellules sont coordonnées par la cellule de crise de la direction d'établissement. À son arrivée, le DMC nommé sur la liste d'aptitude assure le relais de la direction médicale initiée. La zone de triage est organisée. La sécurisation de l'établissement est organisée, ce qui se traduit par une protection assurée face à une attaque menée depuis l'extérieur de l'établissement mais aussi par une sécurisation face à tout individu entrant dans l'établissement, y compris les victimes et accompagnants se présentant spontanément [17]. Le Samu est informé de l'activation du dispositif AMAVI sur l'établissement. Le rappel des personnels est réalisé. Chaque membre du personnel rappelé doit se présenter muni de sa carte professionnelle. Les moyens de communication interne de crise sont déployés. Les décisions sont prises concernant le maintien, l'interruption partielle ou totale de l'activité interventionnelle en cours.

Sous la direction des cellules de pilotage de chaque service impliqué (appelés secteurs), les structures d'accueil des victimes s'organisent pour libérer les places correspondant au flux capacitaire prévu dans le cadre du dispositif AMAVI. Les retours d'expérience formalisés après les attentats de Paris et de Nice rapportent la nécessité de réaliser un zonage au sein de ces secteurs afin de séparer l'accueil AMAVI de l'activité quotidienne [19-22]. Un personnel minimal médical et paramédical reste dédié à la prise en charge des patients issus de l'activité quotidienne et à leur orientation dans les structures d'aval.

Les moyens matériels de l'accueil sont préparés selon une procédure interne, sous forme de kits adaptés au type de victimes accueillies (UA-UR-pédiatrie). Les équipes médicales et paramédicales d'accueil sont identifiées à l'aide de chasubles ou brassards spécifiques. Les structures de réanimation et de soins intensifs libèrent des places en orientant leurs patients en attente de sortie vers les services d'aval.

\section{Organisation des opérations médicales}

\section{Coordination des opérations médicales}

Dès son arrivée sur les lieux, le médecin de la première équipe médicale assure la fonction de premier DSM, et ce, jusqu'à l'arrivée du DSM inscrit sur la liste d'aptitude préfectorale. Son rôle est essentiel : évaluer le volume de renforts, confirmer le choix et l'adresse du ou des point(s) de regroupement des victimes (PRV) et du ou des poste(s) médical avancé (PMA), organiser le tri, la priorisation de prise en charge des victimes et leur évacuation [12].

Concernant les attaques multisites, les retours d'expérience des attentats nationaux et les nombreux exercices de terrain réalisés depuis ont souligné la nécessité de créer une fonction de coordination médicale, supervisant l'ensemble du dispositif médical préhospitalier afin d'organiser le parcours des patients [23]. Nous proposons que cette fonction de coordinateur des opérations médicales soit assurée par le 
chef de service du Samu ou son représentant [24]. Son rôle s'exerce en lien avec le COS et le COPG et en aval avec le DMC. Il a deux missions essentielles au sein du trépied sécurité-secours-santé : coordonner l'ensemble des moyens sanitaires impliqués dans le ou les événements concernés par la tuerie de masse (DSM de site, régulations médicales des Samu, hôpitaux, ARS) et être l'interlocuteur privilégié de l'autorité civile DO concernant le versant santé. À ce titre, il est le garant d'une application cohérente de la doctrine médicale nationale à la crise [16]. Il ne se substitue pas au rôle tactique des DSM et des médecins régulateurs de crises. Son positionnement au moment de la crise dépend du type d'événement, de sa temporalité et de la nécessité de soutien d'une partie ou de toute la filière médicale.

Chaque établissement siège de Samu doit en amont mener une réflexion sur l'alerte et les moyens mis à disposition du chef de service du Samu pour optimiser le délai de son arrivée sur site.

\section{Direction médicale intrahospitalière}

Au sein de l'ES, un DMC est identifié parmi les médecins disponibles habilités à assumer cette fonction. Le DMC a un rôle opérationnel [17]. Une liste d'aptitude à la fonction de DMC est établie par le directeur d'établissement (DE) et le président de la commission médicale d'établissement (CME). La fonction de DMC doit être confiée par le DE à un médecin expérimenté, ayant une pratique de la médecine d'urgence (dans l'idéal un praticien en médecine d'urgence, en anesthésie et/ou réanimation ou en chirurgie d'urgence), reconnu par ses pairs, formé et possédant des aptitudes éprouvées de « leadership ».

Les compétences requises pour occuper cette fonction sont les suivantes : parfaite connaissance de la gestion préhospitalière des secours et des soins, de l'organisation hospitalière, de la gestion des risques NRBCE (en particulier si l'établissement est un établissement de santé de référence) et de la traumatologie d'urgence. Son implication en amont de la crise (relations avec le Samu départemental et le Samu zonal, l'ARS) est un élément structurant.

Le DMC devient au sein de l'ES le correspondant privilégié du Samu en charge de la gestion de l'événement. À ce titre, il informe et reçoit régulièrement des informations du Samu. Il analyse la SSE d'un point de vue médical intrahospitalier. Il évalue les besoins internes permettant d'assurer la réponse à la SSE ainsi que l'activité courante (personnels soignants et médicaux, matériels, plateaux techniques, procédures de soins). Il propose au directeur la réorganisation des soins : modification des activités en cours, réorganisation des équipes, réorganisation des flux de patients (sortants et entrants) et se fait aider dans cette tâche par le président de la CME ou son représentant. Il coordonne/pilote la réponse médicale définie dans le dispositif AMAVI en collaboration avec les coordinateurs de secteurs (médecin tri, coordinateur de la structure d'accueil des urgences (SAU), coordinateur SSPI, chirurgien chef de chaque spécialité, pharmacie, laboratoire, EFS, radiologie, etc.). Il est mobile au sein de l'ES et doit être facilement localisé grâce à des outils de communication. Il délègue les missions en tant que de besoin et travaille en binôme avec un cadre supérieur référent SSE. Chaque établissement doté d'un centre de traumatologie doit en amont mener une réflexion sur l'alerte et les moyens mis à disposition du DMC pour optimiser le délai de son arrivée sur site.

\section{Organisation de la communication opérationnelle}

Ce chapitre aborde la communication nécessaire aux opérations médicales et son articulation avec celle des autres acteurs. La communication avec les médias ou la population relève du rôle exclusif du préfet ou du procureur en lien avec le parquet de Paris.

La communication opérationnelle est nécessaire pour un bon fonctionnement des opérations médicales [25]. Elle doit être formalisée, s'appuyer sur des outils numériques avec une cartographie associée à un géoréférencement [10]. Mal organisée, elle contribue à ralentir et à perturber l'organisation et la prise en charge des victimes. La communication médicale doit être multimodale et interconnectée avec celle des autres acteurs concourant aux missions de sécurité civile [26]. Tous les modes de communication doivent être envisagés en prenant en compte les limitations notamment techniques, les schémas de transmission réglementaires et ceux mis en place par nos partenaires. La voix est actuellement le principal outil de communication (radiophonie, téléphonie, agents de liaison...), mais il est urgent de prévoir des échanges d'informations avec nos différents partenaires en développant et en utilisant des techniques de communication non vocales et sécurisées (informatique, interconnexions logicielles, applications partagées, messageries, etc.) [27].

Pour être opérationnelle, la mise en œuvre doit être simple en utilisant les outils du quotidien. Si des moyens complémentaires sont nécessaires pour la gestion d'une crise, il faut utiliser le plus souvent possible ces moyens de communication de crise dans l'activité courante. Pour chaque typologie de message, un moyen de communication principal doit être défini ainsi qu'un moyen de communication secondaire ou dégradé en cas d'échec du mode de communication principal (indisponibilité d'un réseau ou rétroaction non reçue). En plus du choix des moyens de communication adaptés, une attention doit être portée au choix des terminaux de communication et de leurs accessoires afin qu'ils soient adaptés à leur environnement (robustesse, accessoires tels que des étuis ou des oreillettes pour un poste radio afin de ne pas 
perturber l'environnement, mode haut-parleur ou vidéoprojection d'écran afin de transformer un mode de communication interpersonnelle en communication de groupe...). Enfin, les communications radio doivent idéalement être intégrées dans la téléphonie au casque pour éviter la pollution par le bruit, ce qui existe déjà dans certains Samu.

\section{Régulation médicale}

Compte tenu de leur position stratégique, les SamuCentres 15 doivent être protégés. Les infrastructures techniques doivent être sécurisées et permettre la bascule de l'ensemble des communications sur un système de secours. Ils doivent disposer d'une ligne téléphonique dédiée avec les autres centres de réception d'appels départementaux pour garantir une communication même en cas de saturation des lignes des numéros d'urgence [28]. Nous proposons que ces lignes téléphoniques dédiées soient protégées et enregistrées.

Un ou plusieurs moyens de communication de groupe doivent être disponibles en salle de régulation pour communiquer avec les personnels hospitaliers (information et rappel) ainsi qu'avec les établissements de santé. Ces moyens peuvent être des serveurs téléphoniques (serveurs SMS interactifs et serveurs vocaux de campagne d'appels interactifs) ou complétés par des systèmes de type interphonie diffusant l'information à plusieurs destinataires de manière simultanée. Ces moyens, qui doivent être interfacés avec le logiciel de régulation médicale, doivent pouvoir gérer les retours d'information, permettre de diffuser des informations opérationnelles, mais aussi de l'information générale qui permettra aux acteurs, notamment intrahospitaliers, de s'impliquer en se situant dans l'évolution de la crise. Des messages préformatés doivent être disponibles. L'interactivité de ces systèmes doit permettre de limiter la saturation des capacités de réception (humaines ou techniques). Ils nécessitent la mise en place de procédures de mise à jour régulières des bases de données (annuaires). Une partie de la communication entre la régulation de crise et les établissements de santé peut également être renseignée sur un site Internet dédié dont l'adresse est prédéfinie ou diffusée au moment de l'événement. La régulation médicale du Samu utilise le portail SISamu qui est une extension du SI hospitalier. Le portail SISamu est interfacé avec SI-VIC et par conséquent à SINUS. Il permet de collaborer en situation de crise et d'assurer la régulation médicale de la gestion de crise.

\section{Communication sur site}

Les modalités de communication entre les acteurs concourant sur le terrain sont régies par un organigramme de fonctionnement des réseaux de télécommunication ou ordre particulier de transmission (OPT) qui est préalablement établi par le commandant des systèmes d'information et de com- munication (COMSIC) conformément aux règles fixées par l'Ordre de base départemental des systèmes d'information et de communication (OBDSIC) [29]. Il organise la communication entre les différents acteurs concourant aux missions de sécurité civile et doit être facilement accessible par tous les utilisateurs potentiels. Pour la dimension zonale, un ordre zonal des transmissions doit exister (rôle de l'état-major interministériel de zone), afin d'éviter des doublons de fréquences entre les différents intervenants départementaux présents. Lors de leur élaboration, les Samu doivent exprimer les besoins nécessaires à leur organisation et à la prise en charge des patients, afin que ces besoins soient considérés lors de l'élaboration de ces OPT. Même si pour la communication vocale la communication par Antares doit être privilégiée sur le terrain [30], des moyens de communication satellitaires doivent être disponibles et permettre l'échange de voix et de données [31].

Pour la gestion des victimes, l'objectif est de mettre en place le plus rapidement possible un dispositif qui permette d'organiser la suite des prises en charge. Les fiches médicales de l'avant (FMA) doivent être utilisées. Les systèmes SINUS, SI-VIC et le portail SI-Samu sont interfacés. Ils permettent l'échange et la diffusion de l'information. Le but est d'assurer un dénombrement et une traçabilité des patients du terrain à l'hôpital et au retour au domicile.

Même en l'absence de communication sur le terrain, les données doivent être collectées pour pouvoir les restituer en différé, par exemple par des agents de liaison.

Des infrastructures permettant le support des communications sur le site telles que des relais indépendants ou des bulles Wi-Fi et l'organisation de leur connexion vers l'extérieur doivent être envisagées. Même s'ils restent encore très utilisés, les téléphones portables ne constituent pas un moyen fiable de communication en situation de catastrophe. Le PC de site et la cellule de crise du Samu doivent disposer du flux capacitaire des établissements de santé préétablis pour avoir la capacité d'effectuer sur site la régulation de l'évacuation en cas de défaillance totale des communications [10].

\section{Communication intrahospitalière}

Au même titre qu'en extrahospitalier, la communication intrahospitalière est un outil majeur dont la défaillance conduit à une perte de chance pour les patients. En intrahospitalier, des moyens de communication de crise doivent être mis en place pour assurer d'une part la communication interne à l'établissement entre les cellules de pilotage des secteurs de soins et d'autre part la communication externe entre l'établissement et la direction des soins préhospitaliers (régulation médicale de crise, chef de service du Samu). Le DMC est l'interlocuteur unique pour communiquer avec le Samu. La remontée des informations doit être réalisée de manière pyramidale, des équipes aux cellules de pilotage 
des secteurs de soins, des cellules de pilotage au DMC. Les demandes d'examens tomodensitométriques doivent être centralisées par les cellules de pilotage des secteurs de soins, priorisées et communiquées à la radiologie, afin d'éviter un engorgement de la plateforme d'imagerie médicale. La chaîne de communication interne doit permettre au DMC de connaître la capacité de son établissement à maintenir ou non un flux d'accueil d'UA ou d'UR en connaissant son degré de saturation. La communication interne doit faciliter le parcours des UA et des UR en reliant par des moyens dédiés les zones de tri, de soins, l'imagerie et les cellules de pilotage des secteurs de soins. Elle doit permettre également la diffusion d'informations générales à tous les acteurs de santé de l'établissement, afin qu'ils puissent se situer dans la crise et son évolution. La communication externe doit permettre au DMC d'informer la régulation de crise du degré de saturation de son centre au mieux toutes les 30 minutes. La communication externe doit aussi permettre à la régulation médicale du Samu d'informer le DMC de l'évolutivité de l'événement.

Si des moyens de communication spécifiques à la situation de crise sont mis en place (radio, portatifs avec fréquences dédiées), ils doivent faire l'objet de formations préalables et doivent être intégrés autant que possible dans l'activité quotidienne. Des applications téléphoniques institutionnelles de messagerie de groupe doivent être utilisées. Afin d'utiliser les réseaux de transmission de façon optimale, il est impératif que tous les acteurs de la santé aient reçu une formation initiale et continue sur la communication et l'utilisation des moyens de transmission. Cette formation doit être organisée sous la responsabilité de chaque Samu départemental. Des exercices d'établissement ou préfectoraux ciblés sur la communication doivent être organisés régulièrement. Enfin, il est nécessaire de développer la recherche appliquée dans ce domaine, notamment en créant des bulles de communications permettant d'échanger des consignes opérationnelles entre partenaires de terrain.

\section{Prise en charge médicale en zone d'exclusion}

La zone d'exclusion est initialement définie par le COPG (commandant des opérations de police ou de gendarmerie) qui y exerce l'autorité. Initialement, il s'agit du premier COPG présent sur les lieux. À ce moment de l'événement, si des victimes sont accessibles, les primo-intervenants secouristes ou médecins peuvent procéder aux premières extractions sans attendre la présence des forces de sécurité spécialisées, à la condition expresse que cela soit fait en coordination avec les primo-intervenants des forces de sécurité et qu'il n'y ait pas d'exposition avérée à la menace [32]. En pratique, la phase initiale est une phase de chaos où la confusion est intense. L'évaluation de la menace et sa fixa- tion sont problématiques, puisque les moyens sont encore sous-dimensionnés, et les rumeurs et fausses informations viennent très souvent polluer l'analyse du risque. À son arrivée, le commandant des opérations d'intervention spécialisée (COIS) confirme le choix du zonage.

La zone d'exclusion est artificiellement divisée en deux parties : une zone d'assaut et une zone de reprise. L'accès à la zone d'exclusion est uniquement réservé aux FSI : police et gendarmerie, notamment leurs équipes d'intervention spécialisées. Aucune équipe médicale ni de secours n'est autorisée à entrer en zone d'exclusion. Seul le COPG (ou le COIS par délégation) pourra lever tout ou partie de la zone d'exclusion s'il décrète que les lieux sont sécurisés. Dans l'attente de cette décision, le soutien médical opérationnel de cette zone est assuré par les médecins des forces d'intervention spécialisées (RAID, GIGN, BRI-PP), intégrés aux colonnes d'assaut. Le rôle de ces médecins tactiques est d'assurer le soutien médical des équipes d'intervention et d'organiser l'extraction et le sauvetage des victimes. Ils regroupent ou font regrouper les victimes en nids de blessés puis s'assurent du déplacement de ces victimes vers un ou des points d'extraction des victimes (PEV) sécurisés par les FSI. En fonction de l'évaluation de la menace et du nombre de blessés, le COIS commande la constitution d'un ou de plusieurs PEV au plus près des nids de blessés pour un maximum d'efficacité « secours » à condition que la sécurisation de ces PEV soit possible et assurée. En fonction de la situation (fixation et sécurisation de la zone de contact et du nombre de victimes), le ou les médecins FSI initient les gestes dits de « sauvetage » (garrot, mise en place de pansements compressifs, décompression d'un pneumothorax...) ou délèguent ces gestes aux secouristes des colonnes d'extraction en fonction de leurs compétences [33]. Afin de dimensionner la prise en charge médicale en sortie de la zone d'exclusion, le ou les médecins tactiques effectuent lorsque cela est possible une catégorisation des victimes qui est transmise par anticipation au PC COS et donc au DSM. Toutes les informations utiles au DSM pour anticiper l'arrivée de l'afflux massif de victimes doivent être transmises entre le PC tactique de la FIS, le PC COS et le Samu.

L'extraction des victimes en direction des PRV est assurée par ordre du COS après accord du COIS. Elle n'est possible qu'à la condition que les FSI aient sécurisé temporairement ou définitivement un ou des corridors d'extraction. Ce n'est qu'au PRV que les équipes médicales pourront assurer un tri des victimes. Avant l'entrée au PRV, toutes les victimes sont systématiquement contrôlées par les FSI safety check afin de limiter le risque de surattentat.

Ce dispositif a deux objectifs : sécuriser les lieux afin d'éviter que les personnels de secours ne deviennent des victimes et prendre en charge les blessés le plus rapidement possible pour augmenter leurs chances de survie. La doctrine 
définie veut que la maitrise de la menace prime sur toute action de secours (la police est la force menante).

\section{Triage extrahospitalier}

La prise en charge d'un afflux de blessés repose sur une chaîne ininterrompue de soins qui débute avec le ramassage, la médicalisation sur le terrain, l'évacuation et la prise en charge dans un établissement de santé adapté [26]. Les objectifs sont de sauver le maximum de patients et de sauvegarder leur avenir fonctionnel. Le triage est dynamique et médico-organisationnel. Il permet, à un instant donné, de déterminer le degré de gravité des blessures, de prioriser l'accès aux traitements les plus adaptés et par conséquent de déterminer les modalités d'évacuation [34].

\section{Objectifs de la régulation médicale}

Les retours d'expérience après attentats objectivent une tendance au surtriage des victimes [35-38]. En dépit de la nécessité évidente d'évacuer les victimes dans les délais les plus brefs, il est capital que cette évacuation soit régulée afin de ne pas déplacer les blessés en saturant les possibilités d'accueil des établissements receveurs [18,39]. L'objectif de la régulation médicale est d'adresser chaque patient vers le centre hospitalier public ou privé le plus adapté à ses besoins en termes de soins et de délais de prise en charge.

\section{Organisation du triage}

À l'entrée d'un PRV ou d'un PMA, le triage est effectué par un médecin expérimenté, formé à la médecine de catastrophe et au tri [27]. Il doit impérativement être assisté par un binôme qui n'est pas nécessairement un soignant afin d'assurer les fonctions de secrétariat. Il s'agit d'un acte dynamique qui doit être réévalué à toutes les étapes de la prise en charge [27].

Sur le terrain, les victimes arrivent au PRV ou au PMA après avoir été éventuellement catégorisées par le ou les médecins FSI ou les secouristes des colonnes d'évacuation. Cette catégorisation est faite selon les échelles existantes (valide-non valide/échelle START) [40].

Il existe différents outils de triage extrahospitalier, relativement similaires, classant les patients en quatre ou cinq catégories. La classification du triage médical proposée en médecine d'urgence préhospitalière francophone pour faire face à de nombreuses victimes [41] reste de mise (Fig. 1). Les blessés physiques sont triés de façon binaire en UA et en UR. Au sein des UA, on identifie les urgences extrêmes (EU) pour lesquelles le pronostic vital est engagé en l'absence de prise en charge réanimatoire ou chirurgicale immé- diate et les U1 pour lesquelles le pronostic vital est aussi engagé en l'absence d'une prise en charge réanimatoire ou chirurgicale rapide (délai en heures). Au sein des UR, une répartition classique en deux catégories $\mathrm{U} 2$ et $\mathrm{U} 3$ en fonction du caractère valide ou non de la victime n'a probablement que peu d'intérêt dans le contexte d'une tuerie de masse, la priorité étant l'évacuation des UA. Les autres victimes sont soit impliquées (comprenant les UMP), soit décédées [16]. Le triage en urgence dépassée (UD) concerne une victime qui présente à cet instant des lésions qui à court terme sont incompatibles avec la vie. Les victimes concernées (expectant) seront prises en charge au PMA en attente d'une éventuelle évacuation après les urgences prioritaires (EU et U1), lorsque les moyens disponibles seront suffisants. À ce stade de la prise en charge, une décision de limitation de thérapeutiques n'est pas adaptée [27]. Dans ce contexte, nous proposons que le terme d'UD soit remplacé par le terme d'urgence en attente.

\section{Spécificité pour le tri en pédiatrie}

La prise en charge de nombreux enfants victimes d'attentat comporte des spécificités [42-44]. Comme pour les adultes, le triage définit une priorité à l'évacuation et au traitement [10]. Les particularités anatomiques et physiologiques liées à l'âge impliquent des lésions et des stratégies de prise en charge différentes selon qu'il s'agisse d'enfants de maternelle, du primaire, du collège ou du lycée.

Un triage inadapté peut induire dans un contexte émotionnel exacerbé deux stratégies inappropriées : une sousmédicalisation en cas de sous-évaluation initiale ou au contraire la surmédicalisation d'une UD. Des échelles spécifiques à la pédiatrie de type Jump Start peuvent être utilisées comme aide au triage $[45,46]$. Des aides cognitives doivent être disponibles et rapidement diffusables au sein de chaque Samu [47]. Les auteurs de ce référentiel soulignent l'importance de l'enseignement de la pédiatrie et de l'entretien de ces connaissances pour tous les intervenants en médecine d'urgence.

Une sévérité accrue des lésions et une fréquence élevée des traumatismes crâniens graves en fonction du poids ou de l'âge des enfants ont été rapportées ( $<30 \mathrm{~kg}$; âge : dix ans) [48]. C'est sur ces arguments que la plupart des experts recommandent un accès en centre pédiatrique spécialisé pour les enfants de poids inférieur à $30 \mathrm{~kg}(<30 \mathrm{~kg})$. Cependant, nous pensons qu'en cas d'inadéquation majeure entre le nombre de victimes et les possibilités d'admission en centre de traumatologie pédiatrique spécialisé, la limite devrait être abaissée aux enfants de poids inférieur à $20 \mathrm{~kg}(<20 \mathrm{~kg})$, ce qui correspond aux enfants de moins de six ans scolarisés en maternelle. En dehors des critères d'orientation définis, l'enfant sera dirigé vers un centre de traumatologie adulte. 


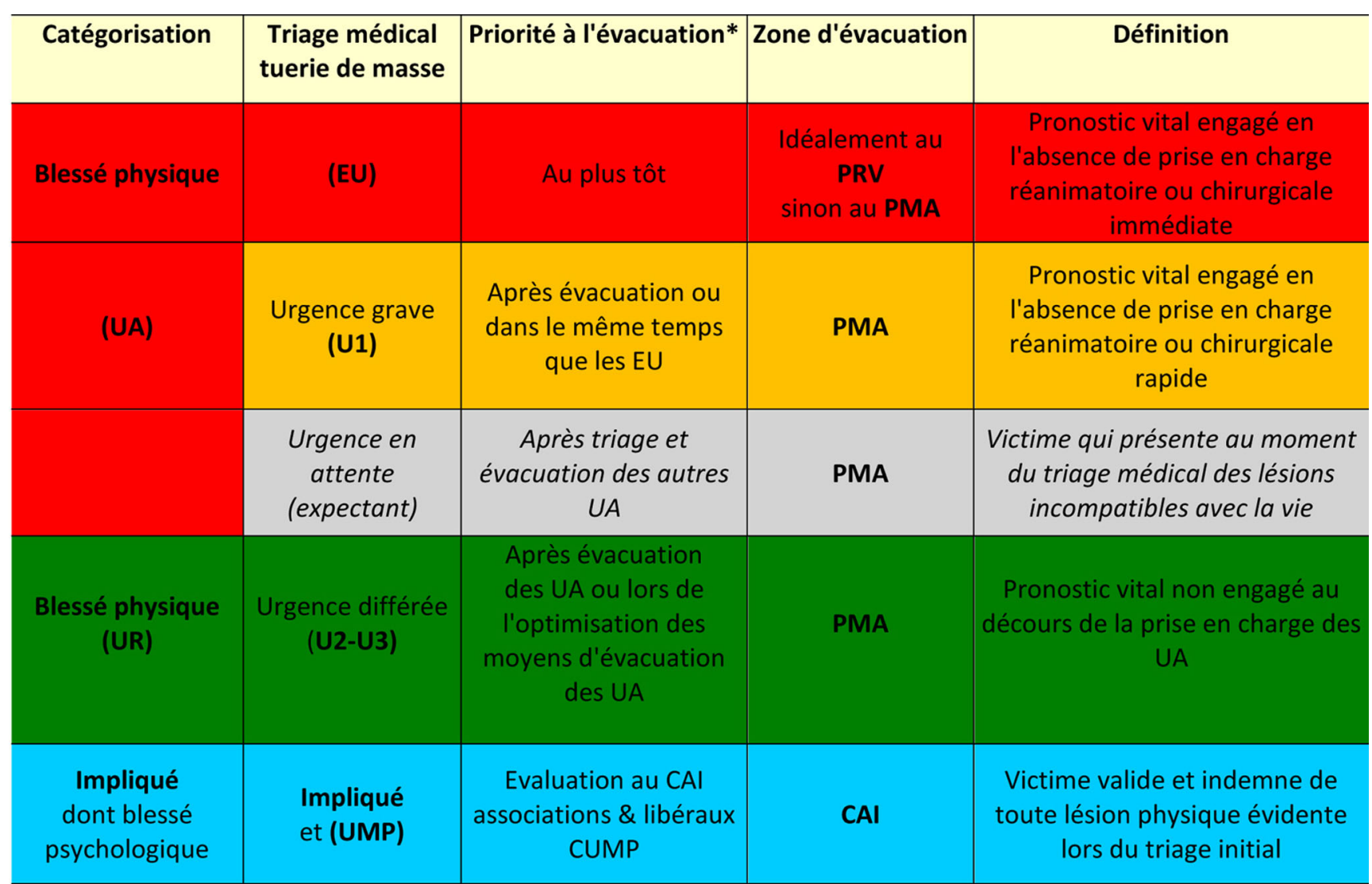

*La cinétique d'une tuerie de masse en milieu civil en France répond à une priorité d'accès aux plateaux techniques spécifiques (réanimation et blocs opératoires).

UA urgence absolue; UR urgence relative; EU : extrême urgence; UMP : urgence médico-psychologique ; PRV point de regroupement des victimes; PMA poste médical avancé ; CUMP : Cellule d'urgence médical et psychologique

Fig. 1 Triage en situation de tuerie de masse pour la priorisation des évacuations sur les destinations hospitalières

En cas de ressources locales spécialisées insuffisantes, deux conduites non exclusives doivent être impérativement définies et formalisées en amont de l'événement :

- la possibilité d'obtenir un renfort préhospitalier (pour aide au tri et à la réanimation) ou hospitalier (par un apport de matériel et d'équipes médicochirurgicales pédiatriques) ;

- les moyens logistiques mis à disposition pour acheminer une partie des victimes vers des centres éloignés adaptés à leur état [24].

Un réel travail d'uniformisation des procédures de prise en charge extra- et intrahospitalière est nécessaire entre les différents centres nationaux, puisqu'il en existe une extrême diversité [49].

\section{PRV, PMA et centre d'accueil des impliqués}

L'objectif principal, compris de tous les primo-intervenants, doit être de prendre en charge les victimes le plus rapidement possible afin de réduire drastiquement le nombre de morts évitables selon de grands principes : regroupement, sécurisation, catégorisation et évacuation. Les soins primaires de DC peuvent être prodigués dès les premières minutes [50,51].

Deux zones permettent en préhospitalier d'optimiser le triage médical, un dénombrement et une priorisation régulée des évacuations : le PRV et le PMA. L'expérience militaire ainsi que les attentats multisites de Paris et de Nice ont montré la pertinence de pouvoir limiter la dispersion des victimes en les regroupant dans des zones sécurisées dès que possible par les FSI. Dans l'esprit de la directive interministérielle [28], la zone d'exclusion étant initialement une hypothèse labile dans le temps et dans l'espace, la dispersion des victimes pouvant être par ailleurs faible ou grande, plusieurs PRV peuvent se créer. Les emplacements des PRV sont de l'ordre de la conduite d'intervention, c'est-à-dire qu'ils sont naturellement fixés par les victimes qui, face à la menace, ont tendance à se regrouper dans des lieux qui paraissent sécurisés. Ces PRV sont confirmés ou éventuellement infirmés dans 
leur pertinence à chaque niveau d'expertise se présentant sur les lieux : FSI, puis soignants non médicaux, enfin médecins. Les renforts médicaux viennent structurer le PRV qui est alors en mesure de se transformer en PMA par définition plus structuré et comportant plus de moyens de prise en charge des lésions. La création de novo d'un PMA se fait en concertation avec le COPG, le COS et le DSM de site.

Chaque PRV ou PMA doit tendre à valider systématiquement cinq critères de pertinence :

- sécurité : à l'abri des menaces selon sa localisation (sécurisation extrinsèque) et la mise en place d'un contrôle à l'entrée (sécurisation intrinsèque) afin de limiter les risques de surattentat ;

- proximité : de la zone de tuerie, in fine en zone de soutien;

- accessibilité : depuis les lieux de la tuerie et permettant une évacuation vers l'arrière dans le respect des axes opérationnels validés par le COPG et le COS ;

- ergonomie : espace, éclairage, chauffage ou climatisation, libre de mobilier ;

- appellation simple unique : répondant à une localisation précise, afin de permettre une identification par tous les intervenants : nom, adresse, point de repère précis. L'utilisation des coordonnées GPS ne semble pas être une solution pratique.

Il faut rapidement et en parallèle mettre en place un centre d'accueil des impliqués (CAI) sous la responsabilité des organisations associatives, par exemple, et en mesure d'accueillir les équipes de la CUMP. Tous ces lieux de regroupement peuvent être multiples. Dans tous les cas, proximité et sécurisation sont indispensables.

Les objectifs d'un PRV sont de regrouper les victimes afin d'initier en sécurité les gestes de DC non réalisés à l'avant et de permettre un dénombrement des victimes par la mise en place de SINUS. En attente du déploiement informatique, la mise en place de SINUS ne doit pas retarder les évacuations [12]. Après mise en place d'un bracelet SINUS à chacune des victimes prises en charge, il suffit alors d'apposer une étiquette du bracelet SINUS sur la FMA de la victime et sur la liste récapitulative du médecin chargé des évacuations. Le PRV permet de prioriser (après réalisation des gestes salvateurs et régulation médicale) l'évacuation des EU directement vers les destinations hospitalières et d'évacuer vers un PMA les autres urgences dans un délai court. Ainsi, les PRV sont soit des zones éphémères, soit des futurs PMA. Un PRV peut initialement ne pas être médicalisé contrairement au PMA qui est placé sous l'autorité d'un médecin.

Le médecin chef du PMA est un médecin urgentiste identifié par le Samu en charge de la gestion de la crise. Le DSM de site confirme la désignation du médecin chef du PMA auprès du COS. Le PMA a pour objectifs de poursuivre le dénombrement des victimes arrivant spontanément ou diri- gées par les primo-intervenants et d'assurer l'accueil des victimes du ou des PRV. Il est organisé en zones (accueil, tri, zones UA/UR, évacuation, dépôt mortuaire). Le PMA permet notamment la prise en charge médicale des EU n'ayant pu être régulées et évacuées directement depuis les PRV.

Dans le cadre particulier d'un attentat, avec des victimes par armes de guerre en milieu civil et des menaces de surattentat, l'expérience montre qu'il est complexe et consommateur de temps d'entrevoir des PRV ou PMA idéaux en structure modulaire par exemple. Les premiers intervenants doivent s'appuyer sur les infrastructures existantes permettant de se focaliser initialement non pas sur la logistique, mais sur la sécurité, le DC et les évacuations régulées des EU. Il est souhaitable de privilégier une entrée et une sortie distinctes, de ne pas laisser entrer d'impliqués (ou les familles) afin de ne pas se faire déborder. Pour les enfants, contrairement aux adultes, la présence d'un des parents à toutes les étapes de la prise en charge préhospitalière semble constituer une plus-value. Afin de faciliter la prise en compte de toutes les victimes, une seule et unique personne effectue la numérotation des FMA, évitant les doublons sur site.

Le CAI a pour objectif d'accueillir les impliqués, mais semblant indemnes de toutes blessures physiques évidentes ou évoquées par le patient. Il peut exister au cœur d'une crise plusieurs CAI qui seront vidés ou bien fusionnés en fonction de son évolution. Le CAI permet une sécurisation des victimes, un dénombrement et l'accès à un soutien matériel ou psychologique. Il est placé idéalement sous la responsabilité de secouristes associatifs, mais peut être renforcé par un ou plusieurs médecins. En cas de décompensation d'une blessure ou d'une pathologie sous-jacente (comorbidités, infarctus de stress, etc.), la proximité du CAI avec le PMA permet de requalifier l'impliqué en blessé et de le rediriger facilement pour évaluation, prise en charge et priorisation d'une évacuation régulée. Il est recommandé qu'un poste d'urgence médicopsychologique (PUMP) soit positionné au sein du CAI afin d'être particulièrement vigilant à la dynamique d'évolution d'une blessure psychique qui peut être absente ou non visible initialement chez un impliqué, à même de décompenser à tout moment et d'imposer alors des soins.

\section{Damage control préhospitalier}

Ce concept dérivé de la pratique médicale militaire a été formalisé au début des années 2000 [52]. Il s'applique aussi bien aux victimes de traumatismes non pénétrants qu'aux victimes de traumatismes pénétrants. L'objectif affiché est une diminution des morts évitables qui sont dans la pratique militaire et civile essentiellement liées à une hémorragie [53-55]. Pour cela, il faut traiter rapidement de façon provisoire ou plus rarement définitive une lésion qui va provoquer la mort à brève échéance et ne pas vouloir restaurer à tout 
prix la normalité des fonctions physiologiques avant l'évacuation et une prise en charge hospitalière [56]. Ce qui impose en pratique :

- de réaliser des gestes d'hémostase [57,58].

La pose d'un garrot nécessite de noter l'heure de pose et de la rendre visible tout en évitant de découvrir le patient pour lutter contre l'hypothermie. L'efficacité et la pertinence du garrot doivent être réévaluées régulièrement à toutes les étapes de la prise en charge. Lorsque le saignement n'est pas accessible à un garrot, un packing doit être utilisé avec une compression externe. Il peut être associé à des pansements compressifs, à des pansements hémostatiques ou à un dispositif externe (ballonnet d'une sonde de Foley par exemple). L'indication d'une contention pelvienne doit être large, en particulier pour les patients victimes d'un traumatisme secondaire à une attaque par un véhicule bélier [59-61] ;

- d'assurer une oxygénation.

L'obstruction des voies aériennes et le pneumothorax suffocant sont respectivement les deuxième et troisième causes de décès évitables en préhospitalier [62]. La libération des voies aériennes supérieures peut être initialement assurée par des gestes simples (évacuation des débris intrabuccaux, subluxation de la mâchoire, mise en place d'une canule de Guedel, position d'attente). C'est secondairement et seulement en situation sécurisée que peut être réalisée une intubation orotrachéale ou une cricothyroïdotomie. Face à une plaie thoracique, un pansement trois côtés est mis en place. En cas de forte suspicion de tamponnade gazeuse associée à une détresse respiratoire aiguë ou hémodynamique, une décompression en urgence devra être réalisée par une exsufflation à l'aiguille ou au mieux par une thoracostomie, complétée par la réalisation d'un pansement trois côtés [63] ;

- de maintenir la perfusion tissulaire.

Il faut préférer chez l'adulte une hypotension permissive avec pour but le maintien d'un contact verbal ou la perception d'un pouls radial et, si la mesure est possible, une pression artérielle systolique voisine de $80 \mathrm{mmHg}$. Un abord veineux périphérique, l'initiation d'un remplissage vasculaire raisonné et l'administration d'une première dose d'acide tranexamique sont souhaitables. L'administration intraveineuse d'adrénaline titrée à $0,1 \mathrm{mg}$ peut être proposée comme solution de sauvetage en l'absence de récupération d'un pouls radial après remplissage initial. En revanche, l'administration en continu de substances vasoactives ou le recours à une transfusion est un choix thérapeutique qui n'a probablement pas sa place à l'exception d'une évacuation retardée pour des contraintes logistiques ;

- de lutter impérativement, de façon active et à toutes les étapes, contre l'hypothermie.
Cette prise en charge dynamique, dans le concept du DC, est associée à une évacuation rapide et régulée vers la structure de soins la plus appropriée au traitement des lésions de la victime. Ce qui impose une approche organisée et un parcours « fléché » de l'évacuation des victimes $[51,64,65]$.

\section{Spécificité pédiatrique}

Les données de la littérature sont insuffisantes pour émettre des recommandations fortes de DC chez l'enfant [66]. Le jeune enfant est plus vulnérable à l'hypothermie et au choc hémorragique compte tenu de son petit volume circulant $[42,47]$. Le concept d'hypotension permissive ne semble pas adapté à ce stade de la prise en charge [44]. La mesure de la pression artérielle n'est pas un bon indicateur des pertes sanguines [67]. La petite taille, la morphologie craniorachidienne et les caractéristiques des voies aériennes majorent la susceptibilité de l'enfant aux traumatismes crâniens et aux asphyxies traumatiques. Nous recommandons d'utiliser des outils d'aide cognitive comme celui proposé par le Samu de Paris [67] ou la réglette de Broselow [46].

\section{Organisation des évacuations}

L'évacuation ne peut se faire qu'après triage et régulation médicale de la destination, quelle que soit la victime $[12,68]$. La priorité d'évacuation est définie après triage. Cependant, en cas d'impossibilité à établir une communication avec la régulation de crise (situations déjà vécues et rapportées au cours de retour d'expérience (RETEX ) d'événements), une régulation médicale anticipée, s'appuyant sur la planification AMAVI, peut être organisée sur site par le premier médecin préhospitalier, prenant de facto la fonction de DSM provisoire. En situation encore plus dégradée et à titre exceptionnel, face à un patient en situation de détresse vitale et à l'absence d'une équipe médicale sur place, un chef d'agrès doit prendre contact avec la régulation médicale du Samu sans délai avant ou, à défaut, au cours du transport, pour orienter la victime vers un établissement hospitalier adapté au niveau de soins requis par le patient [10]. En cas d'inadéquation entre le nombre de victimes et les ressources soignantes disponibles, une évacuation médicalisée individuelle n'est pas réalisable. Il faut organiser des évacuations collectives adaptées à la gravité des patients et aux possibilités d'accueil actualisées de chaque établissement. L'objectif est là aussi de limiter au maximum les morts dites évitables. Les victimes en EU sont évacuées potentiellement en convoi [12]. Elles sont réparties sur les établissements adaptés en fonction d'un schéma d'évacuation préétabli où chaque centre a défini en accord avec la DTARS et le Samu départemental ses volumes capacitaires en cas d'afflux massif de victimes. Les 
vecteurs d'évacuation doivent emprunter un trajet défini en accord avec le COPG et le COS.

En fonction du nombre de victimes et du type de vecteurs sanitaires disponibles, l'évacuation des U1 est faite dans le même temps que l'évacuation des EU ou, dans un deuxième temps, par voie terrestre ou héliportée. Les moyens héliportés (civils ou militaires) seront mobilisés selon le plan zonal prédéfini. Les victimes en urgence relative sont évacuées le plus souvent après les UA ou dans le même temps pour certaines victimes, s'il reste des places disponibles dans les convois.

Dans une configuration optimale, tous les patients évacués doivent avoir été identifiés par la mise en place d'un bracelet SINUS mais, en pratique, à ce stade également, aucune évacuation ne doit être retardée sous prétexte que le patient n'a pas été saisi dans l'outil SINUS. L'interconnexion entre SI-VIC et SINUS permet de venir renseigner le fichier SINUS a posteriori, après admission hospitalière du patient évacué. L'existence d'un patient sera matérialisée dans SINUS par la saisie du numéro et éventuellement sa catégorisation médicale.

\section{Tri à l'entrée de l'établissement de santé}

\section{Pourquoi trier à l'entrée de l'établissement de santé, alors qu'un tri a déjà eu lieu sur le terrain ?}

Quelle que soit la structure hospitalière, l'accueil d'un nombre important de patients dans un délai très court risque de saturer la capacité de prise en charge. La définition d'un afflux saturant reste pourtant relative. Elle dépend de la gra- vité et du nombre de patients, des caractéristiques de la structure hospitalière en termes de taille, de personnels, de spécialités, de moyens techniques, d'architecture et d'occupation des services à un instant donné. Il existe une différence statistiquement significative entre le tri pré- et intrahospitalier [35,69], avec une réelle tendance préhospitalière au surtriage des victimes. Ces données montrent l'importance d'un tri complémentaire réalisé de façon systématique à l'entrée de l'établissement de santé permettant d'adapter les tris initiaux et de détecter une complication survenue pendant la phase d'évacuation [17]. Alors que le tri préhospitalier a pour objectif d'adapter les priorités d'évacuation, le tri intrahospitalier définit l'ordre de passage au bloc opératoire, en imagerie (diagnostique ou interventionnelle), la nécessité d'explorations complémentaires et la mise en condition d'attente ou de transfert (Fig. 2).

Le triage s'appuie sur :

- un bilan lésionnel précis, avec un examen clinique rapide d'un patient déshabillé autant que possible ;

- les conséquences cliniques pour la victime ;

- les données préhospitalières (traitements mis en œuvre en préhospitalier, évolution clinique) si ces données sont disponibles.

\section{Identitovigilance : identification des victimes}

L'inscription dans le système d'information hospitalier (SIH) permet :

- l'enregistrement rapide des victimes quel que soit leur état clinique (en capacité ou en incapacité de décliner leur identité) ;

\begin{tabular}{|c|c|c|c|c|}
\hline $\begin{array}{l}\text { Catégorisation } \\
\text { intrahospitalière }\end{array}$ & $\begin{array}{c}\text { Triage médical tuerie } \\
\text { de masse }\end{array}$ & $\begin{array}{c}\text { Priorité de prise } \\
\text { en charge }\end{array}$ & $\begin{array}{l}\text { Délai de prise } \\
\text { en charge }\end{array}$ & Commentaire \\
\hline \multirow{2}{*}{$\begin{array}{l}\text { Blessé physique } \\
\text { urgence absolue } \\
\text { (UA) }\end{array}$} & $\begin{array}{l}\text { Extrême urgence } \\
\text { (EU) }\end{array}$ & Immédiate & Sans délai & $\begin{array}{l}\text { Pronostic vital engagé en l’absence } \\
\text { de prise en charge immédiate }\end{array}$ \\
\hline & $\begin{array}{l}\text { Urgence grave } \\
\text { (U1) }\end{array}$ & Urgente & < 2 à $6 \mathrm{~h}$ & $\begin{array}{c}\text { Pronostic vital engagé en l'absence } \\
\text { de prise en charge rapide }\end{array}$ \\
\hline \multirow{2}{*}{$\begin{array}{l}\text { Blessé physique } \\
\text { urgence relative } \\
\text { (UR) }\end{array}$} & $\begin{array}{l}\text { Urgences différées } \\
\text { (U2) }\end{array}$ & Différée & < 12 à $18 \mathrm{~h}$ & $\begin{array}{c}\text { Traitement urgent mais pouvant } \\
\text { être différé sans mettre en jeu le } \\
\text { pronostic vital }\end{array}$ \\
\hline & $\begin{array}{l}\text { Urgences différées } \\
\text { (U3) }\end{array}$ & Différée & $<36 \mathrm{~h}$ & $\begin{array}{c}\text { Traitement sans urgence ou } \\
\text { absence d'indication chirurgicale }\end{array}$ \\
\hline \multicolumn{2}{|c|}{$\begin{array}{l}\text { Urgences dépassées } \\
\text { (UD) }\end{array}$} & Dépassée & - & $\begin{array}{l}\text { Lésions graves nécessitant un } \\
\text { traitement lourd et long avec } \\
\text { probabilité limitée de survie }\end{array}$ \\
\hline
\end{tabular}

Fig. 2 Triage intrahospitalier 
- de faire correspondre le numéro d'identification attribué en préhospitalier (SINUS) avec le numéro d'identification attribué par le SIH de l'établissement de santé ;

- l'enregistrement du patient dans le système SI-VIC. Une interface entre SIH et SI-VIC doit être créée afin de faciliter les processus et éviter toute double saisie, source d'erreurs et de perte de temps ;

- la prescription et la réalisation des actes diagnostiques et thérapeutiques requis, la délivrance de produits sanguins labiles.

Afin de répondre aux besoins de rapidité d'admission des patients en gravité «UA » ou de nombreuses victimes dans l'incapacité de décliner leur identité, il est recommandé de réaliser une édition anticipée du matériel nécessaire à la préadmission sous $\mathrm{X}$ d'un nombre adapté à la capacité d'accueil du centre. Cette procédure permet d'avoir accès sans délai à un numéro identifiant permanent du patient (IPP), aux étiquettes et bracelets d'identification et à l'initiation des soins, sans saisie informatique à l'arrivée du patient. Le bracelet d'identification doit être apposé à chaque victime. L'étiquette doit être collée sur le bracelet d'identification lors de son admission. Un recueil de correspondance entre le numéro d'identification des victimes attribué en préhospitalier (SINUS), le numéro d'identification IPP de l'établissement de santé et les identités présumées doit être mis en place.

Une fiche d'identification des victimes non communicantes et décédées doit être remplie [17]. La description faite doit faciliter les démarches d'identification des victimes en recueillant : l'identité présumée si elle est connue et son mode de révélation (déclarée à l'arrivée, présente sur des effets personnels), un signalement morphologique classique complet avec l'indication de signes distinctifs : cicatrices, piercing, tatouage, marques cutanées, malformations..., la description des vêtements et l'inventaire des effets personnels. La prise d'un cliché de face (avec un appareil photographique spécifiquement dédié) associé au numéro d'IPP peut être proposée [70].

Le circuit d'admission hospitalier doit être réfléchi pour éviter à un patient d'entrer en zone de soins sans admission et identification préalables. Une cellule dédiée d'admission en SSE doit être mise en place.

\section{Où trier ?}

Une sécurisation des flux de victimes doit être organisée en amont du tri. Une zone d'entrée unique des victimes dans l'établissement est déterminée. Le lieu du tri doit être anticipé : grand hall aux abords des SAUV par exemple [17]. La zone de triage se situe nécessairement en amont de la chaîne de soins. Les travaux d'architecture des ES devront anticiper un tel espace en cas de besoins SSE.
Le tri intrahospitalier est réalisé dès l'admission des victimes, juste après leur enregistrement administratif dans le système d'information de l'ES et sur la plateforme SI-VIC. Nous répétons ici la nécessité de créer une interface unique entre les deux systèmes.

Trois zones de soins sont identifiées : pour les UA, pour les UR et pour les impliqués. La répartition des victimes au sein de ces zones est assurée par un tri d'entrée.

Il est mis en place une signalétique pour permettre l'accès aux différentes zones de soins.

La zone traitant les UA sera localisée idéalement à proximité des plateaux interventionnels (blocs opératoires, radiologie). La zone traitant les UR pourra être localisée au sein du SAU. La zone traitant les impliqués sera localisée à distance des zones de soins des UA et des UR.

Chaque secteur de soins est piloté par une cellule composée d'un responsable médical et d'un responsable paramédical. Le parcours des patients doit être conçu selon une politique de " marche en avant " afin de conserver le plus longtemps possible une capacité d'accueil pour les nouvelles victimes.

\section{Quels outils pour trier?}

Un tableau permet de noter au fur et à mesure l'identification des patients, le triage préhospitalier, la description des blessures et de l'état clinique, la catégorisation après triage hospitalier et la destination retenue. Ce tableau, consultable par tous, en particulier par le DMC, est une importante source d'information. Il s'agit d'un tableau informatisé dédié partagé ou à défaut d'un support de type tableau blanc mobile. Conçu en amont, il fait partie du plan blanc. Au cours du temps, ce tableau est mis à jour avec les informations provenant des médecins responsables des différentes zones de prise en charge. S'il n'est pas informatisé, avant d'être effacé, ce tableau doit être photographié à des fins de mémorisation.

\section{Qui trie?}

Le concept de tri s'articule autour de deux points essentiels que sont d'une part l'appréciation des grandes fonctions vitales et d'autre part l'évaluation des lésions et de leur potentiel évolutif défavorable. Il permet ainsi une prise rapide de décision fondée sur la catégorisation définie pour la victime.

Il convient de rassembler autour du patient deux praticiens expérimentés se partageant cette double évaluation des grandes fonctions et des lésions, au cours d'une évaluation rapide, complète, intégrant par leur expérience le potentiel évolutif.

Le couple médecin anesthésiste-réanimateur et chirurgien peut constituer un tel binôme, comme peut l'être également 
un binôme médecin urgentiste et chirurgien. Le chirurgien doit préférentiellement être un chirurgien viscéral ou thoracique avec de l'expérience dans la prise en charge des traumatismes graves. Il apprécie la gravité clinique des lésions, les saignements peropératoires attendus, les besoins chirurgicaux, le délai d'attente possible. Le médecin anesthésisteréanimateur ou urgentiste apprécie la réserve physiologique, les besoins transfusionnels, les abords veineux et artériels et l'ensemble des mesures non chirurgicales.

$\mathrm{Au}$ fur et à mesure de la prise en charge des blessés, et parce qu'il est continu dans le temps, ce triage pourra être assuré par le médecin anesthésiste-réanimateur ou le médecin urgentiste seul en cas d'afflux mobilisant le chirurgien au bloc opératoire.

Le médecin désigné responsable du tri est identifié grâce à une chasuble. Une fiche de poste « médecin trieur» doit être disponible et doit comporter :

- le rôle du médecin trieur ;

- l'organisation de la zone de tri ;

- ses interactions avec les autres acteurs et leurs numéros de téléphone ;

- les données qu'il doit communiquer, leur délai et à qui ;

- les zones d'orientation des patients ;

- les particularités pédiatriques (poids ou âge limite pour l'orientation vers le secteur adulte ou pédiatrique);

- un listing d'aide au tri.

\section{Organisation de la logistique des soins hospitaliers}

Une équipe médicale et paramédicale doit pouvoir être dédiée à chaque UA, selon un modèle classique d'accueil en traumatologie lourde [71]. Un trauma leader est identifié pour chaque UA. Dans les structures susceptibles d'accueillir de nombreuses UA, le responsable du secteur UA assure un rôle de supervision des soins. Il confirme et priorise les choix diagnostiques et thérapeutiques. Il rend compte au DMC. Une fois la prise en charge diagnostique et thérapeutique réalisée, l'UA est dirigée vers une structure d'aval de réanimation ou de soins intensifs et l'équipe réinjectée pour la prise en charge des futures victimes. Un support visuel (tableau) doit permettre le suivi des UA.

Les circuits d'examens complémentaires, de mise à disposition des produits sanguins, de réapprovisionnement de pharmacie, de mise à disposition du matériel médical (scopes, respirateurs de transport, obus d'oxygène, pousseseringues) et de remise en condition des aires d'accueil sont identifiés et centralisés sur la zone d'UA. Des personnels soignants sont dédiés à chaque circuit.
Rappel des personnels, gestion des personnels et organisation des relèves

Le DMC évalue les besoins médicaux et soignants en fonction de l'intensité de la crise et de son caractère évolutif ou non, sur la base des informations communiquées par le Samu. Il transmet son analyse à la cellule de crise hospitalière $(\mathrm{CCH})$ pour mise en œuvre des procédures de rappel [72]. Dans ce cas, la CCH doit être informée des demandes formulées, des réponses obtenues et des effectifs rappelés, comme des effectifs disponibles.

Le rappel doit se faire au moyen de dispositifs automatisés paramétrables, permettant de gérer l'appel, la réponse de l'appelé et la mise à disposition de la $\mathrm{CCH}$ et du $\mathrm{DMC}$ de ces informations sous la forme d'un tableau de bord. Il faut éviter la réponse des personnels par un appel afin de ne pas saturer les capacités téléphoniques.

Le dimensionnement du rappel doit tenir compte du caractère évolutif et de la cinétique de la crise, de sorte que $\mathrm{du}$ personnel soit mis en réserve pour assurer les relèves.

La question de la relève doit être posée précocement en fonction de la charge de travail afin d'anticiper et d'organiser les relèves. Les personnels rappelés doivent porter une carte professionnelle munie d'une photographie permettant de les identifier.

Les personnels soignants sont affectés à des postes adaptés à leur connaissance de l'urgence et de la fonction. Certains professionnels étrangers à l'établissement peuvent participer à la prise en charge des victimes, au titre de « collaborateurs occasionnels » à la condition qu'ils aient la capacité à exercer la fonction (carte professionnelle) et aient reçu l'autorisation de la $\mathrm{CCH}$.

Il est important d'avoir clarifié en amont la situation des personnels médicaux ou paramédicaux exerçant une activité volontaire dans les SDIS (Service départemental incendie et secours). En cas d'activité partagée entre le secteur hospitalier et le volontariat au SDIS, un rappel par les deux secteurs peut émaner. Pour les personnels hospitaliers volontaires physiquement postés au SDIS, il est admis qu'ils poursuivent leur mission côté SDIS. En revanche, les personnels hospitaliers volontaires sapeurs-pompiers non postés physiquement au SDIS doivent rester à la disposition de l'employeur hospitalier $[28,73]$.

\section{Damage control intrahospitalier et prise en charge médicale}

\section{Principes}

Le DC a comme objectif de conjuguer étroitement les actions de réanimation et de chirurgie, selon une temporalité spécifique pour le meilleur pronostic vital et fonctionnel du 
patient. En pratique, ses deux volets en sont la réanimation DC et la chirurgie DC [74]. Les objectifs du premier sont :

- d'arrêter les hémorragies (vérifier le garrot préhospitalier, limiter le saignement avec un objectif de pression artérielle peu élevée (pression artérielle systolique à $80 \mathrm{mmHg}$ à l'exception d'un traumatisme crânien associé) ;

- de lutter contre les troubles de coagulation (en limitant le remplissage vasculaire par recours aux amines vasoactives, en luttant contre l'hypothermie, en utilisant l'acide tranexamique comme antifibrinolytique) ;

- de transfuser précocement (concentré de globules rouges, plasma décongelé ou lyophilisé, plaquettes, fibrinogène).

Conjointement à ces actions de réanimation, les gestes du chirurgien obéissent aux mêmes règles d'efficacité et de parcimonie au cours de la chirurgie DC [75-77]. Ces actions ont pour objectif de sauver la vie en restaurant la physiologie et non l'anatomie :

- en contrôlant les foyers hémorragiques (hémostase);

- en prévenant les infections (coprostase);

- en fermant les brèches aériennes (aérostase).

Ces actions chirurgicales visent préférentiellement un traitement en deux temps afin de pouvoir reprendre précocement les actions de réanimation. Ce traitement chirurgical initialement écourté (une heure) est décidé d'un commun accord avec le médecin anesthésiste-réanimateur soit d'emblée devant l'état clinique du patient, soit en cours de chirurgie devant l'étendue des lésions prolongeant déraisonnablement l'acte au regard des actions de réanimation à entreprendre. C'est la même chirurgie dans une stratégie différente, pour sauver la vie et la fonction. Ce principe de traitement, bénéfique au plan individuel, peut l'être aussi collectivement en garantissant une plus grande disponibilité des blocs opératoires face à une situation évolutive [78]

\section{Indications individuelles de damage control}

Les indications de DC sont d'abord liées à l'état clinique du patient. Un patient présentant une altération des grandes fonctions vitales relève d'une réanimation initiale, d'une procédure de chirurgie écourtée pour permettre les meilleures chances de rétablissement d'une homéostasie avant la poursuite de la prise en charge chirurgicale dans un deuxième temps. C'est le cas pour un patient présentant une coagulopathie $(\mathrm{TP}<50 \%)$, une hypothermie (température $\left.<35{ }^{\circ} \mathrm{C}\right)$, une acidose lactique $(\mathrm{pH}<7,20)$, un état de choc, une transfusion massive.

Les indications de DC peuvent également être chirurgicales : un saignement abondant difficilement accessible nécessite un packing pour obtenir une hémostase ; un geste vasculaire chirurgical trop long à réaliser peut être temporairement différé par l'utilisation de shunt vasculaire ; une technique chirurgicale non réalisable en un temps (résections intestinales avec rétablissement de la continuité différé), un risque de syndrome du compartiment abdominal sont des indications reconnues de laparostomie dans le cadre d'un DC chirurgical.

\section{Existe-t-il des indications collectives de damage control ?}

En cas d'afflux massif, en particulier en cas de saturation du bloc opératoire, une décision d'indication collective de DC chirurgical peut être prise afin d'assurer à tous les blessés un accès au traitement chirurgical dans le délai approprié. Cette décision, concordante avec les principes du triage, revient au DMC et procède d'une indication collective plutôt qu'individuelle. Ainsi, les réparations lésionnelles d'orthopédie en un seul temps, nécessitant plusieurs heures, seront réalisées en deux temps ou orientées vers une exofixation, alors même que l'état clinique du patient ne le justifie pas. Cette conduite permet de libérer les blocs opératoires plus rapidement et donc de rester disponibles pour d'autres arrivées de blessés. Elle impose une discipline collective de la communauté médicale au cours de la crise. Cette décision doit être tracée et communiquée à tous les acteurs. Une fois levée, elle est également tracée. Les principes du DC pour la réanimation et pour la chirurgie doivent faire l'objet d'une description dans le plan blanc de l'établissement. Les acteurs doivent s'y former et idéalement s'y entraîner au cours d'un exercice « plan blanc » dans leur établissement.

\section{Rôle de la cellule d'urgence médicopsychologique}

Le psychiatre coordinateur (psychiatre référent de la CUMP départementale impactée ou le cas échéant de la CUMP régionale ou de la CUMP zonale) et son adjoint administratif (cadre de l'ARS) sont désignés par l'ARS.

Ils sont chargés [79] :

- d'assurer l'accueil et la coordination des équipes médicopsychologiques (MP) mobilisées en renfort ;

- de mettre en place une équipe d'appui logistique chargée d'assurer la mise en œuvre des PUMP, le planning, le transport, les repas et l'hébergement des équipes MP de renfort ;

- d'assurer l'interface avec l'ARS, la préfecture, les collectivités territoriales, le centre d'accueil des familles (CAF) et les associations d'aide aux victimes ;

- d'assurer la coordination des autres acteurs contribuant à la prise en charge MP ;

- d'organiser en lien avec les établissements de santé concernés la prise en charge MP des personnels et des professionnels de santé mobilisés dans l'événement. 


\section{Cellule d'urgence médicopsychologique}

Dès qu'elle est activée, la CUMP :

- organise la mise en place d'un ou de plusieurs PUMP installés au CAI ;

- s'assure de la traçabilité des victimes prises en charge dans le ou les PUMP, en utilisant le logiciel SI-VIC (à ce titre, la présence d'un personnel administratif doit être anticipée) ;

- prodigue des soins MP immédiats aux victimes et à toutes les personnes impliquées dans l'événement, y compris les professionnels de santé et les sauveteurs [80];

- demande une évacuation après régulation par le Samu des victimes nécessitant une hospitalisation vers les établissements de santé ;

- délivre un certificat médical descriptif des lésions MP aux victimes prises en charge au PUMP et leur remet la note d'information conformément au modèle national ;

- organise si besoin, en lien avec le Samu, une réponse MP téléphonique (PUMP téléphonique) afin d'apporter une réponse adaptée aux victimes ;

- dispense des soins postimmédiats aux patients le nécessitant et les oriente, le cas échéant, vers un dispositif de suivi adapté ;

- coordonne, dans le domaine qui la concerne, l'action des autres acteurs contribuant à l'aide et au soutien des victimes.

Un coordonnateur PUMP est prévu sur chaque intervention, il donne les directives à son équipe et constitue l'interlocuteur privilégié des partenaires et des décideurs.

La cellule interministérielle d'aide aux victimes (CIAV) met en place un CAF dont la mission essentielle est l'accueil, le recensement et l'information des familles qui recherchent des proches potentiellement victimes de l'événement. La prise en charge de ces familles doit toutefois être assurée dans le cadre d'un PUMP attenant au CAF. Le responsable de PUMP assure l'interface avec le responsable du CAF en liaison avec le psychiatre coordinateur et son adjoint administratif.

Il est prévu un accueil téléphonique des familles, géré par la CIAV.

\section{Formations initiales et continues}

Ces formations concernent l'ensemble des professionnels médicaux et paramédicaux, quels que soient leur lieu et leur mode d'exercice. L'histoire montre qu'une tuerie de masse peut concerner l'ensemble du territoire national [1]. Les professionnels de santé doivent être individuellement formés à la prise en charge de victimes d'une tuerie de masse [72]. Dans les formations délivrées, les dimensions organisation- nelles, d'aide à la prise de décision en urgence, les aspects psychologiques et techniques auxquels ces professionnels seront confrontés doivent être appréhendés [81]. Les personnels des CUMP doivent être formés aux consignes de sécurité individuelles.

\section{Formations universitaires et institutionnelles}

\section{Diplôme d'études spécialisées}

Le diplôme d'études spécialisées (DES) de médecine d'urgence (DESMU) intègre un enseignement lié aux SSE. Les principes du tri en médecine de catastrophe et le déclenchement de la CUMP sont enseignés pendant la phase socle. Les principes de la médecine de catastrophe, de la gestion des urgences collectives et des SSE sont enseignés pendant la phase d'approfondissement. Il s'agit d'une première approche en termes de gestion d'urgences collectives [82].

Le DES de médecine intensive et réanimation (DESMIR) et le DES d'anesthésie-réanimation (DESAR) intègrent dans leur programme un enseignement sur les SSE dans le cadre des enseignements transversaux universitaires (modules 15.3, 15.4).

\section{Capacité ou diplôme universitaire de médecine de catastrophe}

Plusieurs universités proposent une capacité ou un diplôme universitaire (DU) de médecine de catastrophe. Cet enseignement se déroule sur un an et concerne les médecins et infirmiers. Il a pour objectif de préparer les soignants à intervenir sur les lieux de sinistres, d'accidents ou de catastrophes naturelles, technologiques ou de société, entraînant de nombreuses victimes. Cet enseignement forme les professionnels à participer à l'organisation des secours et des soins médicochirurgicaux « de masse » et permet d'acquérir une culture de la gestion de la catastrophe et du risque qui est différente de celle mise en œuvre lors des soins d'urgence quotidienne.

Diplômes universitaires de prise en charge de traumatisés

Différents DU existent. Ils peuvent être à destination des médecins urgentistes ou anesthésistes-réanimateurs (DU de prise en charge des traumatisés sévères) ou à destination des chirurgiens (DU de traumatologie viscérale).

\section{Attestations de formation aux gestes et soins d'urgence}

Les centres d'enseignement des soins d'urgence (CESU), les unités de formation et de recherche des UFR, le SSA délivrent une attestation de formation aux gestes et soins d'urgence (AFGSU) de niveaux 1 et 2 , dans laquelle un module est consacré aux principes d'organisation sanitaire en SSE. 
L'instruction DGOS du 26 juin 2017 présente les axes prioritaires 2018 pour le développement des compétences des personnels des établissements relevant de la fonction publique hospitalière. L'un des axes retenus est la formation des professionnels de santé aux gestes et soins d'urgence et aux situations sanitaires exceptionnelles. Il est important d'inciter les directions des établissements de santé à développer la formation à l'AFGSU spécialisée SSE (dix modules), intégrant notamment la formation des professionnels de santé à la prise en charge des victimes d'attentats (formation « DC »), à l'intervention MP pour les personnels des CUMP et aux risques NRBCE [83]. Elle doit s'intégrer au plan de formation des établissements de santé et participer au développement professionnel continu des professionnels de santé $[8,17,83]$. La formation des professionnels de santé à la prise en charge des blessés par armes de guerre est inscrite au plan de formation des établissements de santé ayant un rôle défini dans le volet AMAVI du dispositif ORSAN. Elle repose sur l'AFGSU spécialisée.

\section{Formations spécifiques}

Des actions de formation doivent être créées à l'intention des professionnels libéraux. Certaines fonctions nécessitent une formation spécifique afin d'harmoniser les pratiques, pouvant être exercées par des personnels d'institutions différentes (DSM, DMC). Les médecins inscrits sur les listes d'aptitudes à la fonction de DSM devraient bénéficier d'une formation spécifique. Concernant les fonctions de coordination des opérations médicales assurées par le chef de service du Samu et les fonctions de DMC, il est nécessaire de définir et de labéliser une ou des formations universitaires ou institutionnelles.

\section{Formations internationales}

Au-delà des formations internationales préparant à une prise en charge protocolée des traumatisés graves, il existe des formations ou techniques pédagogiques qui intègrent le contexte spécifique de multiples victimes et l'utilisation de la simulation pour la gestion de SSE. C'est le cas notamment du « Medical Response to Major Incidents » (MRMI), formation par la simulation de la réponse sanitaire à une situation d'exception destinée à la totalité de la chaîne des secours et des soins médicaux intégrant également la chaîne de commandement, dont le déploiement adapté à la France est en cours, en lien avec les sociétés savantes nationales concernées.

\section{Plans de secours et exercices}

\section{Diffusion et connaissance des plans de secours}

L'efficience des professionnels de santé passe par une bonne connaissance des dispositifs ORSEC, ORSAN et du plan blanc de leur établissement. Il est primordial que ces plans soient largement diffusés à l'ensemble des personnels de l'ES, notamment lors de chaque mise à jour. Ces plans doivent être facilement consultables et leur localisation connue de tous, aussi bien en version numérique que papier. Afin d'assurer une appropriation de ces plans par les personnels de l'établissement de santé, il faut développer des actions de formation du personnel par petits groupes permettant les échanges et le questionnement, avec des supports pédagogiques adaptés (diaporama, vidéos, serious game), ainsi que des exercices de dimension variable selon les objectifs fixés. La mise en place de fiches actions adaptées aux différentes fonctions et accessibles facilement est un élément permettant de faciliter l'organisation de la réponse des secours. Les personnels des directions hospitalières doivent bénéficier d'une formation à la gestion des SSE, comme celle délivrée par l'EHESP $[84,85]$.

\section{Exercices}

\section{- Réglementation}

Les exercices permettent de tester les dispositions générales et spécifiques du dispositif opérationnel et peuvent impliquer la participation périodique de la population. Les différents textes de loi prévoient la réalisation chaque année d'un ou de plusieurs exercices ou entrainements associant les acteurs du système de santé, permettant d'évaluer le caractère opérationnel des dispositifs ORSEC et ORSAN et de valider les formations aux soins et gestes d'urgence (FGSU) SSE $[8,86]$.

Le ministre chargé de la Sécurité civile assure la synthèse et la diffusion au niveau national des retours d'expérience réalisés sous l'autorité du représentant de l'État après tout recours au dispositif ORSEC, qu'il s'agisse d'un événement réel ou d'un exercice [85]. Ces RETEX doivent être utilisés pour la formation du personnel.

Tous les établissements de santé, universitaires ou non universitaires, quelle que soit leur zone géographique d'implantation, doivent s'organiser afin de libérer du temps médical et paramédical pour la pratique régulière d'exercices cadres ou terrain [87].

\section{- Types d'exercices existants}

Il existe deux formes d'exercices à réaliser selon les objectifs visés et les ressources disponibles (humaines, matérielles, financières, temporelles, etc.).

\section{Exercices cadres (ou d'état-major ou sur table)}

Les nombreux objectifs visés par l'établissement ne peuvent être abordés en une seule fois et au cours d'un seul exercice. Il est possible de réaliser des exercices partiels et segmentés afin de valider ou d'ajuster certaines parties d'un plan. 
L'exercice cadre permet aussi de tester des objectifs particuliers ou de travailler sur des objectifs difficiles à tester comme la montée en puissance d'un plan, le commandement des différents services, la communication interservices [24]. La préparation et la réalisation d'un exercice partiel peuvent s'inscrire dans une démarche progressive d'appropriation de la « doctrine exercice » et constituer ainsi une première étape avant l'organisation d'exercices de plus grande envergure. L'avantage indéniable des exercices cadres est leur moindre coût par rapport à un exercice de terrain.

\section{Exercices de terrain}

Ils permettent de tester les plans dans leur ensemble, de l'alerte à l'hospitalisation des patients ou à la sortie de crise. Ils peuvent également ne concerner que le préhospitalier ou l'intrahospitalier. C'est l'enchaînement des différentes phases du scénario qui est observé. Le décorticage d'une phase en particulier doit être étudié préalablement au cours d'un exercice partiel (cadre ou terrain). Il est important de s'exercer aux mesures à prendre en début de crise, particulièrement celles concernant le partage des informations et la communication en interne et en externe avec les multiples intervenants sur site ou en intrahospitalier.

Un exercice de terrain permet de tester en grandeur réelle des phases très importantes comme l'alerte et l'information des populations, les délais d'acheminement et de mise en œuvre des moyens extra- ou intrahospitaliers, le délai de mise en œuvre d'un périmètre de bouclage, la mise à l'abri ou l'évacuation de la population, les interfaces et la communication entre les différents services, entre les établissements de santé ou en intrahospitalier. Lors d'un exercice terrain, l'ensemble des acteurs qui seraient sollicités en situation réelle doivent être conviés à participer. Dans le cadre particulier d'un exercice « tuerie de masse », l'articulation et la communication avec les forces de l'ordre doivent faire partie des objectifs. L'exercice terrain permet aussi d'y associer la population, contribuant ainsi à son éducation et à sa sensibilisation face au danger. L'exercice général peut combiner des séquences de terrain et cadres, mais toutes les phases doivent être abordées pour vérifier la cohérence de leur enchaînement et la pertinence globale du plan.

Liens d'intérêts : les auteurs déclarent ne pas avoir de lien d'intérêt.

\section{Références}

1. Carli P, Pons P (2018) Introduction générale. Agressions collectives par armes de guerre. Conduites à tenir pour les professionnels de santé. http://solidarites-sante.gouv.fr/IMG/pdf/Agressions-collectives.pdf (Dernier accès le 2 août 2018)

2. Assemblée nationale (2016) Rapport fait au nom de la commission d'enquête relative aux moyens mis en œuvre par l'État pour lutter contre le terrorisme depuis le 7 janvier 2015. http://www.assem- blee-nationale.fr/14/pdf/rap-enq/r3922-t1.pdf (Dernier accès le 16 juillet 2018)

3. Lagadec P (2012) Du risque majeur aux mégachocs. In: Les cahiers de préventique. Préventique Éd., Paris, pp 1-224

4. Salomon J, Courreges A (2018) Avant-propos. Agressions collectives par armes de guerre. Conduites à tenir pour les professionnels de santé. http://solidarites-sante.gouv.fr/IMG/pdf/Agressionscollectives.pdf (Dernier accès le 2 août 2018)

5. Carli P, Pons F, Levraut J, et al (2017) The French emergency medical services after the Paris and Nice terrorist attacks: what have we learnt? Lancet 390:2735-8

6. Callaway DW (2018) A review of the landscape: challenges and gaps in trauma response to civilian high threat mass casualty incidents. J Trauma Acute Care Surg 84:S21-S7

7. Haute Autorité de santé (2016) Guide méthodologique. Élaboration de recommandations de bonnes pratiques. https://www. has-sante.fr/portail/upload/docs/application/pdf/2011-01/guide_methodologique_recommandations_pour_la_pratique_clinique. pdf (Dernier accès le 21 septembre 2018)

8. République française (2016) Décret $\mathrm{n}^{\circ}$ 2016-1327 du 6 octobre 2016 relatif à l'organisation de la réponse du système de santé (dispositif " ORSAN ») et au réseau national des cellules d'urgence médicopsychologique pour la gestion des situations sanitaires exceptionnelles. https://www.legifrance.gouv.fr/affichTexte. do? cidTexte $=$ LEGITEXT000033217065\&dateTexte $=2018908$ (Dernier accès le 21 septembre 2018)

9. Foucher S, Le Loch JB, Desbrest A, et al (2018) Catastrophe avec nombreuses victimes en milieu urbain : plan blanc et difficultés de gestion des places hospitalières. Med Armees 46:213-24

10. Lesaffre X, Tourtier JP, Violin Y, et al (2017) Remote damage control during the attacks on Paris: lessons learned by the Paris Fire Brigade and evolutions in the rescue system. J Trauma Acute Care Surg 82:S107-S13

11. Ministère de la Justice (2018) Comment alerter la police ou la gendarmerie en cas d'urgence ? https://www.justice.fr/fiche/alerter-police-gendarmerie-cas-urgence (Dernier accès le $1^{\text {er }}$ septembre 2018)

12. Ernouf C, Vivien B (2018) Organisation des secours et des soins médicaux. Agressions collectives par armes de guerre. Conduites à tenir pour les professionnels de santé. http://solidarites-sante. gouv.fr/IMG/pdf/Agressions-collectives.pdf (Dernier accès le 2 août 2018)

13. Ministère des Affaires sociales et de la Santé (2014) Guide d'aide à l'organisation de l'offre de soins en situations sanitaires exceptionnelles. https://sofia.medicalistes.fr/spip/IMG/pdf/Guide_d_aide_ a_1_organisation_de_1_offre_de_soins_en_situations_sanitaires exceptionnelles.pdf (Dernier accès le 21 septembre 2018 )

14. Turner C, Lockey DJ, Rehn M (2016) Pre-hospital management of mass casualty civilian shootings: a systematic literature review. Crit Care 20:362

15. Vivien B, Carli P (2009) Le plan rouge : de la théorie à la pratique. In: Journées scientifiques de la Société française de médecine d'urgence. Elsevier Masson Éd., Paris, pp 9-55

16. Premier ministre (2017) Instruction interministérielle 5979/SG du 10 novembre 2017 relative à la prise en charge des victimes d'actes de terrorisme. http://circulaire.legifrance.gouv.fr/pdf/2017/11/ cir_42753.pdf (Dernier accès le 19 septembre 2018)

17. Daban JL, Raux M (2018) Accueil hospitalier. Agressions collectives par armes de guerre. Conduites à tenir pour les professionnels de santé. http://solidarites-sante.gouv.fr/IMG/pdf/Agressionscollectives.pdf (Dernier accès le 2 août 2018)

18. Aylwin CJ, König TC, Brennan NW, et al (2006) Reduction in critical mortality in urban mass casualty incidents: analysis of triage, surge, and resource use after the London bombings on July 7, 2005. Lancet 368:2219-25 
19. Orban JC, Quintard H, Ichai C (2017) ICU specialists facing terrorist attack: the Nice experience. Intensive Care Med 43:683-5

20. Carles M, Levraut J, Gonzalez JF, et al (2016) Mass casualty events and health organization: terrorist attack in Nice. Lancet 388:2349-50

21. Gregory T, Bihel T, Guigui P, et al (2016) Terrorist attacks in Paris: surgical trauma experience in a referral center. Injury 47:2122-6

22. Borel M, Le Saché F, Pariente D, et al (2016) Retour d'expérience des attentats du 13 novembre 2015. Rôle d'un hôpital disposant d'un centre de traumatologie. Ann Fr Med Urgence 6:22-30

23. Braun F, Carli P (2017) Coordinateur des opérations médicales : « le DSM 2.0 ». Jeurea 29:208-11

24. Braun F, Benner P (2018) L'aide médicale d'urgence dans les territoires : organisation de la réponse. Agressions collectives par armes de guerre. Conduites à tenir pour les professionnels de santé. http://solidarites-sante.gouv.fr/IMG/pdf/Agressions-collectives.pdf (Dernier accès le 2 août 2018)

25. Philippe JM, Brahic O, Carli P, et al (2016) French Ministry of Health's response to Paris attacks of 13 November 2015. Crit Care 20:85

26. Witkowski J (2018) Préface. Agressions collectives par armes de guerre. Conduites à tenir pour les professionnels de santé. http:// solidarites-sante.gouv.fr/IMG/pdf/Agressions-collectives.pdf (Dernier accès le 2 août 2018)

27. Puidupin A, Cazes N, Michaloux M, et al (2018) Triage et régulation des afflux. Agressions collectives par armes de guerre. Conduites à tenir pour les professionnels de santé. http://solidarites-sante.gouv.fr/IMG/pdf/Agressions-collectives.pdf (Dernier accès le 2 août 2018)

28. République française (2015) Circulaire interministérielle $\mathrm{n}^{\mathrm{o}}$ DGOS/R2/DGSCGC/2015/190 du 5 juin 2015 relative à l'application de l'arrêté du 24 avril 2009 relatif à la mise en œuvre du référentiel portant sur l'organisation du secours à personne et de l'aide médicale urgente. https://solidarites-sante.gouv. fr/fichiers/bo/2015/1507/ste_20150007_0000_0045.pdf (Dernier accès le 21 septembre 2018 )

29. République française (2009) Arrêté du 23 décembre 2009 relatif à l'ordre de base national des systèmes d'information et de communication de la sécurité civile. https://www.legifrance.gouv.fr/eli/ arrete/2009/12/23/IOCE0931439A/jo/texte (Dernier accès le 19 septembre 2018)

30. Ministère des Affaires sociales et de la Santé, ministère de l'Intérieur (2017) Instruction interministérielle du 4 mai 2016 relative à la préparation de situations exceptionnelles de type attentats multisites. http://circulaire.legifrance.gouv.fr/pdf/2017/03/cir_41996. pdf (Dernier accès le 21 septembre 2018)

31. Khorram-Manesh A (2016) Europe on fire; medical management of terror attacks. New era and new considerations. Bull Emerg Trauma 4:183-5

32. Abriat A, Cazes N, Lara A, et al (2018) Gestes secouristes sur le lieu de l'attaque. Agressions collectives par armes de guerre. Conduites à tenir pour les professionnels de santé. http://solidarites-sante.gouv.fr/IMG/pdf/Agressions-collectives.pdf (Dernier accès le 2 août 2018)

33. Service médical du RAID (2016) Tactical emergency medicine: lessons from Paris marauding terrorist attack. Crit Care 20:37-8

34. Julien H, Allonneau A, Bon O, Lefort H (2018) Aspects actuels du triage, du combat à la catastrophe, essai de synthèse. Med Armees 46:197-206

35. Frykberg ER (2002) Injury, medical management of disasters and mass casualties from terrorist bombings: how can we cope? J Trauma 53:201-12

36. Gutierrez de Ceballos PJ, Turégano Fuentes F, Perez Diaz D, et al (2005) Casualties treated at the closest hospital in the Madrid, March 11, terrorist bombings. Crit Care Med 33:S107-S12
37. Singer AJ, Singer AH, Halperin P, et al (2007) Medical lessons from terror attacks in Israel. J Emerg Med 32:87-92

38. Li GQ, Hou SK, Yu X, et al (2015) A descriptive analysis of injury triage, surge of medical demand, and resource use in an university hospital after 8.12 Tianjin Port Explosion, China. Chin J Traumatol 18:314-9

39. Frykberg ER, Tepas JJ (1988) Terrorist bombings. Lessons learned from Belfast to Beirut. Ann Surg 208:569-76

40. START Triage (2018) Simple triage and rapid treatment. http:// www.start-triage.com/ (Dernier accès le 6 septembre 2018)

41. Noto R, Huguenard P, Larcan A (1987) Tri et catégorisation des victimes. In: médecine de catastrophe. Masson Éd., Paris, pp 239-51

42. Alix-Séguin L, Lodé N, Orliaguet G, et al (2017) Et si c'était des enfants? Adaptation de la prise en charge médicale en cas d'attentats terroristes avec de nombreux enfants victimes. Arch Pediatr 24:280-7

43. Duracher C, Vergnaud E, Meyer P, et al (2016) Damage control appliqué à la pédiatrie. J Anrea 2:247-53

44. Bouchut JC, Peguet O, Chassery C, et al (2018) Prise en charge préhospitalière pédiatrique en situation d'urgence collective : spécificités ou vraie doctrine pédiatrique ? J Anrea [in press]

45. Romig LE (2002) Pediatric triage: a system to JumpSTART your triage of young patients at MCIs? JEMS 27:52-8

46. Leplatois T, Brezzo C, Horlaville S, et al (2017) Afflux massif de victimes pédiatriques. In: Congrès Sfar, Journées Urgences vitales. Sfar Éd., Paris, pp 1-12

47. Lemoine S, Chabernaud JL, Ernouf C, Tourtier JP (2016) Prise en charge préhospitalière de victimes pédiatriques multiples en situation d'urgence collective. Arch Pediatr 23:1109-11

48. Jaffe DH, Peleg K; Israel Trauma Group (2010) Terror explosive injuries: a comparison of children, adolescents and adults. Ann Surg 251:138-43

49. Mortamet G, Lode N, Roumeliotis N, et al (2018) Disaster preparedness in French paediatric hospitals 2 years after terrorist attacks of 2015. Arch Dis Child 1-6 [in press]

50. Ministère de l'Intérieur (2017) Tuerie de masse : réponse opérationnelle des services d'incendie et de secours. http://www.cmub. $\mathrm{fr} / \mathrm{wp}$-content/uploads/2017/04/Note-de-doctrine-operationnelletuerie-de-masse-DGSCGC-2017-03-20.pdf (Dernier accès le 21 septembre 2018)

51. Malgras B, Prunet B, Lesaffre X, et al (2017) Damage control: concept and implementation. J Visc Surg 154:S19-S29

52. Johnson JW, Gracias VH, Schwab CW, et al (2001) Evolution in damage control for exsanguinating penetrating abdominal injury. J Trauma 51:261-9

53. Howard JT, Kotwal RS, Santos-Lazada AR, et al (2018) Reexamination of a battlefield trauma golden hour policy. J Trauma Acute Care Surg 84:11-8

54. Oyeniyi BT, Fox EE, Scerbo M, et al (2017) Trends in 1,029 trauma deaths at a level 1 trauma center: impact of a bleeding control bundle of care. Injury 48:5-12

55. Jouffroy R, Travers S (2018) Damage control préhospitalier. Agressions collectives par armes de guerre. Conduites à tenir pour les professionnels de santé. http://solidarites-sante.gouv.fr/ IMG/pdf/Agressions-collectives.pdf (Dernier accès le 2 août 2018)

56. Tourtier JP, Palmier B, Tazarourte K, et al (2013) The concept of damage control: extending the paradigm in the prehospital setting. Ann Fr Anesth Reanim 32:520-6

57. Kragh JF, Walters TJ, Baer DG, et al (2009) Survival with emergency tourniquet use to stop bleeding in major limb trauma. Ann Surg 249:1-7

58. Schroll R, Smith A, McSwain NE, et al (2015) A multiinstitutional analysis of prehospital tourniquet use. J Trauma Acute Care Surg 79:10-14 
59. Duranteau J, Asehnoune K, Pierre S (2015) Recommandations sur la réanimation du choc hémorragique. Anesth Reanim 1:62-74

60. Rossaint R, Bouillon B, Cerny V (2010) Management of bleeding following major trauma; an updated European guideline. Crit Care 14:R52

61. Massaloua D, Ichai C, Mariagea D, Baqué P (2018) Terrorist attack in Nice. The experience of general Surgeons. J Visceral Surg [in press]

62. Eastridge BJ, Mabry RL, Seguin P, et al (2012) Death on the battlefield (2001-2011): implications for the future of combat casualty care. J Trauma Acute Care Surg 73:431-7

63. Société française d'anesthésie et de réanimation, Société française de médecine d'urgence (2015) Traumatisme thoracique : prise en charge des 48 premières heures. Anesth Reanim 3:272-87

64. Butler F (2003) Tactical combat casualty care: combining good medicine with good tactics. J Trauma 54:S2-S3

65. Pons P, Carli P (2018) Principes du damage control. Agressions collectives par armes de guerre. Conduites à tenir pour les professionnels de santé. http://solidarites-sante.gouv.fr/IMG/pdf/Agressions-collectives.pdf (Dernier accès le 2 août 2018)

66. Schauer SG, April MD, Hill GJ, et al (2018) Prehospital interventions performed on pediatric trauma patients in Iraq and Afghanistan. Prehosp Emerg Care 22:624-9

67. Telion C, Khen-Dunlop N (2018) Spécificités pédiatriques du damage control. Agressions collectives par armes de guerre. Conduites à tenir pour les professionnels de santé. http://solidarites-sante.gouv.fr/IMG/pdf/Agressions-collectives.pdf (Dernier accès le 2 août 2018)

68. République française (2016) Instruction interministérielle $\mathrm{n}^{\mathrm{o}} 5853 / \mathrm{SG}$ du 13 avril 2016 relative à la prise en charge des victimes d'actes de terrorisme. http://circulaires.legifrance.gouv. fr/pdf/2016/04/cir_40765.pdf (Dernier accès le 21 septembre 2018)

69. Wang NE, Pirrotta E, Vogel L (2017) Transfer and under-triage patterns of seriously injured children in California (2005-2013). Ann Emerg Med 70:S135

70. GRIVES (2017) Guide de référence d'identitovigilance en région PACA. http://grives-paca.org/outils/charte-r\%C3\%A9gionale-etguide-r\%C3\%A9ference (Dernier accès le 25 août 2018)

71. Committee on trauma (2014) Resources for optimal care of the injured patient. American College of Surgeons Ed., Chicago

72. Mantz B, Pelée de Saint Maurice G (2018) Organisation et logistique hospitalières. Agressions collectives par armes de guerre. Conduites à tenir pour les professionnels de santé. http://solidarites-sante.gouv.fr/IMG/pdf/Agressions-collectives.pdf (Dernier accès le 2 août 2018)

73. Ministère de l'Intérieur, de l'Outre-mer et des Collectivités territoriales, ministère de la Santé, de la Jeunesse, des Sports et de la Vie associative (2008) Organisation du secours à personne et de l'aide médicale urgente. http://www.ladocumentationfrancaise.fr/ rapports-publics/084000373/index.shtml (Dernier accès le 24 septembre 2018)

74. Le Noël A, Merat S, Ausset S, et al (2011) Le concept de damage control resuscitation. Ann Fr Anesth Reanim 30:665-78

75. Blackbourne LH (2008) Combat damage control surgery. Crit Care Med 36:S304-S10

76. Roberts DJ, Bobrovitz N, Zygun DA, et al (2015) Indications for use of damage control surgery and damage control interventions in civilian trauma patients: a scoping review. J Trauma Acute Care Surg 78:1187-96

77. Roberts DJ, Ball CG, Feliciano DV, et al (2017) History of the innovation of damage control for management of trauma patients: 1902-2016. Ann Surg 265:1034-44

78. Boddaert G, Hornez E, Barbier O (2018) Damage control chirurgical. Agressions collectives par armes de guerre. Conduites à tenir pour les professionnels de santé. http://solidarites-sante. gouv.fr/IMG/pdf/Agressions-collectives.pdf (Dernier accès le 2 août 2018)

79. Rerbal D, Prieto N, Vaux J, et al (2017) Organisation et modalités d'intervention des cellules d'urgence médicopsychologique. Recommandations de la Société française de médecine d'urgence (SFMU) en collaboration avec l'Association de formation et de recherche des cellules d'urgence médicopsychologique et la Société française de psychotraumatologie (AFORCUMPSFP). Ann Fr Med Urgence 7:410-2

80. Martinez LM, Colas MD, Cremniter D, et al (2018) Prise en charge médicopsychologique des victimes. Agressions collectives par armes de guerre. Conduites à tenir pour les professionnels de santé http://solidarites-sante.gouv.fr/IMG/pdf/Agressions-collectives.pdf (Dernier accès le 2 août 2018)

81. Mouchet A, Bertrand C (2018) Décider en urgence au SamuCentre 15. Octarès Éd., Toulouse

82. République française (2017) Arrêté du 21 avril 2017 relatif aux connaissances, aux compétences et aux maquettes de formation des diplômes d'études spécialisées et fixant la liste de ces diplômes et des options et formations spécialisées transversales du troisième cycle des études de médecine. https://www.legifrance. gouv.fr/eli/arrete/2017/4/21/MENS1712264A/jo (Dernier accès 21 septembre 2018)

83. République française (2017) Instruction $\mathrm{n}^{\mathrm{o}}$ DGOS/RH4/ DGCS/4B/2017/211 du 26 juin 2017 relative aux orientations retenues pour 2018 en matière de développement des compétences des personnels des établissements mentionnés à l'article 2 de la loi $\mathrm{n}^{\mathrm{o}}$ 86-33 du 9 janvier 1986 portant dispositions statutaires relatives à la fonction publique hospitalière. http://circulaire.legifrance.gouv.fr/pdf/2017/07/cir_42389.pdf (Dernier accès le 21 septembre 2018)

84. Ministère des Affaires sociales et de la Santé (2013) Instruction $n^{\circ}$ DGS/DUS/CORRUSS2013/274 du 27 juin 2013 relative à l'organisation territoriale de la gestion des situations sanitaires exceptionnelles. https://solidarites-sante.gouv.fr/fichiers/bo/2013/ 13-08/ste_20130008_0000_0176.pdf (Dernier accès le 22 septembre 2018)

85. Ministère des Affaires sociales et de la Santé (2014) Instruction $\mathrm{n}^{\mathrm{o}}$ DGS/DUS/SGMAS/2014/153 du 15 mai 2014 relative à la préparation du système de santé à la gestion des situations sanitaires exceptionnelles. https://solidarites-sante.gouv.fr/fichiers/bo/ 2014/14-06/ste_20140006_0000_0050.pdf (Dernier accès le 22 septembre 2018 )

86. République française (2004) Loi n ${ }^{\circ} 2004-811$ du 13 août 2004 de modernisation de la sécurité civile. https://www.legifrance.gouv. fr/affichTexte.do?cidTexte=JORFTEXT000000804612 (Dernier accès le 22 septembre 2018)

87. Gauss T, Cook F; the Traumabase Group (2017) Keep calm... and prepare. BMJ Qual Saf 26:786-87 